\title{
Solvable model of gas production decline from hydrofractured networks
}

\author{
M. Marder (1)* \\ Department of Physics, The University of Texas at Austin, Austin, Texas 78712, USA \\ Behzad Eftekhari 10 \\ Enthought Energy Solutions, 1502 Sawyer Street, Suite 232 Houston, Texas 77007, USA
}

Tadeusz W. Patzek $\odot$

Petroleum Engineering Research Center, King Abdullah University of Science and Technology, Thuwal 23955, Saudi Arabia

(Received 30 June 2021; revised 27 September 2021; accepted 17 November 2021; published 9 December 2021)

\begin{abstract}
We address questions that arose from studying gas and oil production from hydrofractured wells. Does past production predict the future? This depends on deducing from production as much as possible about the plausible geometries of the fracture network. We address the problem through a solvable model and use kinetic Monte Carlo and Green's function techniques to solve it. We have three main findings. First, at sufficiently long times, the production from all compact fracture networks is described by a universal function with two scaling parameters, one of which is the diffusivity of unbroken rock $\alpha$ and the second of which is a parameter $\mathcal{V}_{\text {ext }}$ with units of volume. Second, for fracture networks where the power-law distribution of fracture spacings falls below a critical value (and this appears to be the case in practice), early-time production always scales as one over the square root of time. Third, the diffusivity $\alpha$ that sets the scale for late-time production is inherently difficult to estimate from production data, but the methods here provide the best hope of obtaining it and thus can determine the physics that will govern the decline of unconventional gas and oil wells.
\end{abstract}

DOI: 10.1103/PhysRevE.104.065001

\section{INTRODUCTION}

Hydrofracturing liberates gas and oil from mudstones, often called shales, that form layers hundreds or thousands of meters deep and 30 to $100 \mathrm{~m}$ thick [1,2]. Hundreds of thousands of these "unconventional" wells have now been drilled, and the U.S. natural gas supply depends upon them $[3,4]$. The extraction process requires creating a fracture network through a subsurface stimulation process involving explosives, pressurized water, and sand [5]. The resulting fracture networks extend over a distance on the order of a kilometer in one direction, and on the order of $200 \mathrm{~m}$ in the perpendicular horizontal direction. Gas diffusion in unfractured shales is very slow; the diffusivity $\alpha$ is on the order of $10^{-8} \mathrm{~m}^{2} / \mathrm{s}$ [6]. However, once gas reaches the fractures it travels rapidly to a wellbore and from there up to the surface. We will mainly refer to gas production throughout this article, but the same physical picture and equations apply to oil as well [7].

The shapes and connectivity of fracture networks that lead to production are poorly known. Microseismic imaging provides clues based upon acoustic emissions detected during the fracturing process $[8,9]$, but the sound emission is not likely to provide an accurate map of the fractures. Rapidly moving brittle fractures do not have to generate strong acoustic emissions, and noise from the grinding of rock faces in shear does not have to correspond to an opening crack. More clues have come from diagnostic wells [10] and from ingenious new acoustic

*marder@chaos.utexas.edu techniques [11]. These techniques are expensive, rarely employed, and provide only partial geometrical information.

Despite the inevitable complexity of fracture networks created underground, one can make a great deal of progress by imagining that the fracture network adopts the parallel plate geometry shown in Fig. 1 [6]. Two important quantities characterizing underground fracture networks can be extracted from the time history of gas production. These two quantities are $\mathcal{M}$, the stimulated original gas in place, and the interference time $\tau=d^{2} / \alpha$, where $\alpha$ is the diffusivity of gas and $2 d$ is the spacing between the plates. The stimulated original gas in place is the original gas density $\rho_{0}$ times the stimulated reservoir volume $\mathcal{V}$. One of our goals here is to understand why this model performs so well given that the geometry on which it is based is so simple.

Our larger goal, however, is to find if the time dependence of gas production contains enough information that it is possible to extract details about the geometry of the fracture network. At first the answer appears to be yes. The time dependence of gas production is given by a sum over exponential terms from the solution of an eigenvalue problem. This operation can in principle be inverted, the eigenvalues in principle extracted, and these then in principle provide detailed signatures of the geometry.

However, the inverse problem that moves from production history to network geometry is ill-posed. Large changes in the network produce almost no change in production. We demonstrate this not through a strict mathematical method, but through careful physically motivated analysis in two different production regimes. 

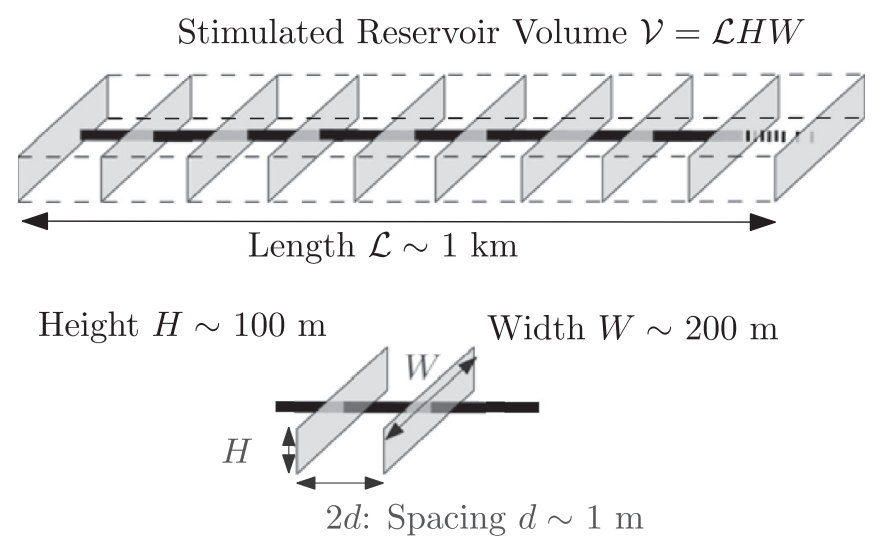

FIG. 1. The geometry of the parallel plate model [6] consists of a horizontal well with a uniform array of planar hydrofractures. The cuboid volume that contains the well and the hydrofractures is known as the stimulated reservoir volume. Gas transport in this model involves diffusion of gas pressure in the interior of the stimulated volume and normal to the hydrofractures. Gas transport from the exterior is neglected.

The first regime is the long-time regime. At sufficiently long times, gas production for all compact fracture networks is described to very high accuracy by two numbers. The first of these is the diffusivity $\alpha$ of unfractured rock, which sets the physical scale. The second number $\mathcal{V}_{\text {ext }}$ is the volume within an equivalent absorbing square boundary; all details about the network other than the volume of the square become irrelevant.

The second regime is the early-time regime. Here the situation is a little more complicated. Suppose the probability of finding a region where the spacing between nearby fractures is $d$ goes as $P\left(d^{2}\right) \propto d^{-2 \beta}$. For all networks where the exponent $\boldsymbol{\beta}$ is less than the critical value of $1 / 2$, the early-time behavior is the same as the parallel plate model in Fig. 1. This condition applies to all model systems we studied. Thus we obtain an explanation for why the parallel plate geometry matches experimental data so well, but we also obtain discouraging news about the prospects of deducing geometry from production. At both early and late times information about all networks collapses down into two early-time and two late-time parameters. It is only during the crossover period that some more information can be extracted. We describe methods to do so, although even in idealized theoretical settings we have not gotten them to work as well as we originally hoped. We show that by including the power law $\beta$ in our models we can improve their ability to fit production histories in ways that improve the modeling accuracy according to standard statistical criteria. This process may or may not withstand the test of use with real noisy data. In any event, we believe our methods point to how to extract the most possible physically reliable information from production data.

An obvious and vexing sign that the inversion problem is ill-posed appears in the parallel plate model. The first clue about geometry one ever sees in production data is a cumulative production curve bending below a line going as $t^{1 / 2}$. In the parallel plate model, this happens on the timescale $\tau=d^{2} / \alpha$ (Fig. 1). The interference time $\tau$ is the time it takes the pressure from neighboring fracture planes to diffuse to the halfway point between the fractures. The problem here is that $\tau$ is compatible with an infinite number of values of $d^{2}$ and $\alpha$, so long as their ratio is conserved. This degeneracy breaks down at late times when flow is dominated by gas coming from unbroken rock. But how long does one need to wait for that late-time information? How clear is the signal?

The setting in which we address all these questions is an exactly solvable model. The expectation, as for other simple models in physics, is that this will make it possible to understand qualitative ideas, that the conclusions will have a universal character, and that the hosts of details we omit turn out not to make a large difference. The particular model we define is similar to some that have been studied before. At first it appears most similar to diffusion limited aggregation [12], because it features diffusive motion on a lattice, where walkers arrive at a surface. However, in our problem, the absorbing surface is static and does not change as time progresses. Therefore, there is actually a greater formal affinity with the problem of electron transport in disordered solids, and we will draw on the methods developed for that context throughout this paper. We present two methods to solve the model. The first method is a kinetic Monte Carlo approach. This was the first method we tried. Then we developed a second method that draws on lattice Green's functions to obtain exact time-dependent solutions, at the cost of greater formal complexity. We have published a preliminary account of the second method [13]. Here we provide an improved formalism that delivers answers around 100 times faster, we provide many more details of how the method operates, and then we use the method to answer the questions we have posed.

While our work here occurs in a completely idealized setting, we were motivated by and will eventually return to the application of these ideas to field data. A similar version of this approach to long-time production behavior was already applied to field data in $[14,15]$. The fact that using power-law distributions of fractures can improve fits to data was found previously [16]. To explore the implications of single-well production models for field-wide production estimates see [4] and references therein.

In Sec. II we describe the kinetic Monte Carlo approach and the conclusions we drew from it. In Sec. III we summarize results from Green's function methods that permit exact solutions. In Sec. IV we explore the relation between fracture network geometry and production. In the course of developing the applications, we demonstrate universality of long-time production, show how to extract information about the power-law structure of the fracture network, use it to make predictions about future production, and show why this procedure is ultimately unsatisfying. Section V summarizes our conclusions. Details concerning the Green's function formalism are found in four Appendixes.

\section{KINETIC MONTE CARLO}

\section{A. Model}

The fracture network in hydrofractured shales is contained in a finite region around the well commonly referred to as the stimulated reservoir volume. As sketched in Fig. 1, the length 


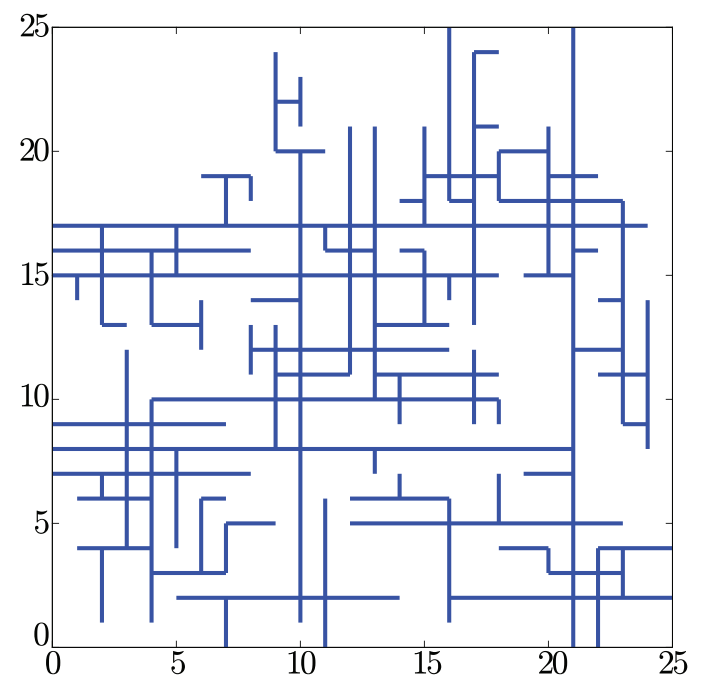

FIG. 2. Geometry of our Monte Carlo model, with a stimulated reservoir volume (fractures in blue) embedded in a fracture-free matrix (white). The precise structure of the fracture network comes from a geologically constrained percolation model described in detail in $[17,18]$. In the model, fracture length results from a power-law probability distribution characterized by the exponent $e$. The density of the fracture network is controlled by the filling fraction $p$, defined as the ratio of the broken lattice bonds to the total number of bonds.

$\mathcal{L}$ of the well in the horizontal direction is kilometers, while the height $H$ of the shale layer is $30-100 \mathrm{~m}$. This is why all the models in this paper are defined in two dimensions. Some qualitative phenomena might require a three-dimensional explanation, but we focus here on what can be learned from assuming that the essential phenomena are contained in two dimensions.

Our first numerical experiments were carried out on systems such as a $25 \times 25$ square lattice with periodic boundaries shown in Fig. 2. The bonds between adjacent lattice sites can be broken or left intact, to mimic fracture or unbroken rock. The blue lines depict fractures, and when gas located in the white space diffuses to a fracture, it is absorbed and removed from the system. The fractures are viewed as infinitely thin, and the gas diffuses in a continuous plane.

Our fracture network comes from a lattice percolation model based on observations of fractures in geological formations [17]. Fractures in the model lie along two primary orthogonal directions. In accord with a large body of geological research, the length of the fractures is distributed according to a power law of exponent $e$, such that the cumulative density function for length $l$ is given by [19,20]

$$
F(l)=\frac{l^{-e}-l_{\min }^{-e}}{l_{\max }^{-e}-l_{\min }^{-e}} .
$$

Here $l_{\min }$ and $l_{\max }$ are the minimum and maximum lengths: $l_{\min }$ is the same as the lattice spacing; $l_{\max }$ is infinite. In geological fracture systems, $e$ has been shown to vary typically between 0.8 to 2.2 , with a mode at 1.2. The geological data are for rocks fractured by natural processes, which need not produce the same power laws as hydrofracturing. However, in the absence of better information we begin here. Fracture density in the model is controlled by the filling fraction $p$, defined as the ratio of broken lattice bonds to the total number of bonds. Our fracture network is the spanning cluster for the percolation problem fully defined by $e$ and $p$ [17].

Given the network in Fig. 2, we examine the diffusion problem involving transport of ideal gas to an absorbing network of infinitely conductive fractures. Initial gas saturation in the model is uniform, and we assume homogeneous transport properties for the unbroken lattice. We are interested in the rate of mass transport to the fracture network.

We simulate diffusive transport using a Monte Carlo scheme involving independent random walks. We populate the lattice with a uniform initial density of random walkers and let each walk continue until the walker is absorbed on the fracture network. The probability density function for the walker arrival time, which one can construct by considering sufficient numbers of walks, gives the time history of the mass transport rate.

We determine each hop by sampling the displacement distribution $\phi(x)$ and the waiting time distribution $\psi(t)$ for Brownian motion:

$$
\phi(x)=\frac{1}{\sqrt{2 \pi} \sigma} \exp \left(-\frac{(x-\mu)^{2}}{2 \sigma^{2}}\right),
$$

where $\sigma \ll$ lattice spacing and $\mu=0$ (no drift), and

$$
\psi(t)=\frac{1}{t^{*}} \exp \left(-\frac{t}{t^{*}}\right) .
$$

In practice we set $t^{*}=1$ and $\sigma=0.1$. This corresponds to a diffusion constant for the two-dimensional problem of $D=$ $\sigma^{2} /\left(2 t^{*}\right)=0.005$.

\section{B. Validation}

We validated our Brownian walk simulation by comparing with an analytical solution [6] for flow to parallel plates in one dimension. The geometry is given in Fig. 3(a). We show in Sec. III D 2 that the difference between the one-dimensional solution for plates and two-dimensional solution for squares is insignificant. Particles start at a randomly chosen point in the interior and diffuse until they hit a boundary.

Starting with a uniform initial density of random walkers, we simulate Brownian motion until all walkers are absorbed on the fractures. Next, we fit the solution for flow into squares to the probability density function for the walkers' arrival time and obtain the scaled rate vs time plot presented in Fig. 3(b). There is a good agreement between the analytical result [Eqs. (28) and (29)] and Monte Carlo calculations.

\section{Results}

We now apply the Monte Carlo scheme of Sec. II A to several fracture networks. Figure 4 shows the production rate for the fracture network presented in Fig. 2. At early times, the rate declines in accord with the parallel plate solution [6]: the rate initially declines as $1 / \sqrt{t}$ and transitions to an exponential at about $\tilde{t}=1$. The $1 / \sqrt{t}$ decline emerges from the diffusion of low gas pressure from the fractures through what appears to be a semi-infinite reservoir at early times. Next, the rate begins to decline as a power law and maintains this behavior over approximately a decade in scaled time. The 
(a)
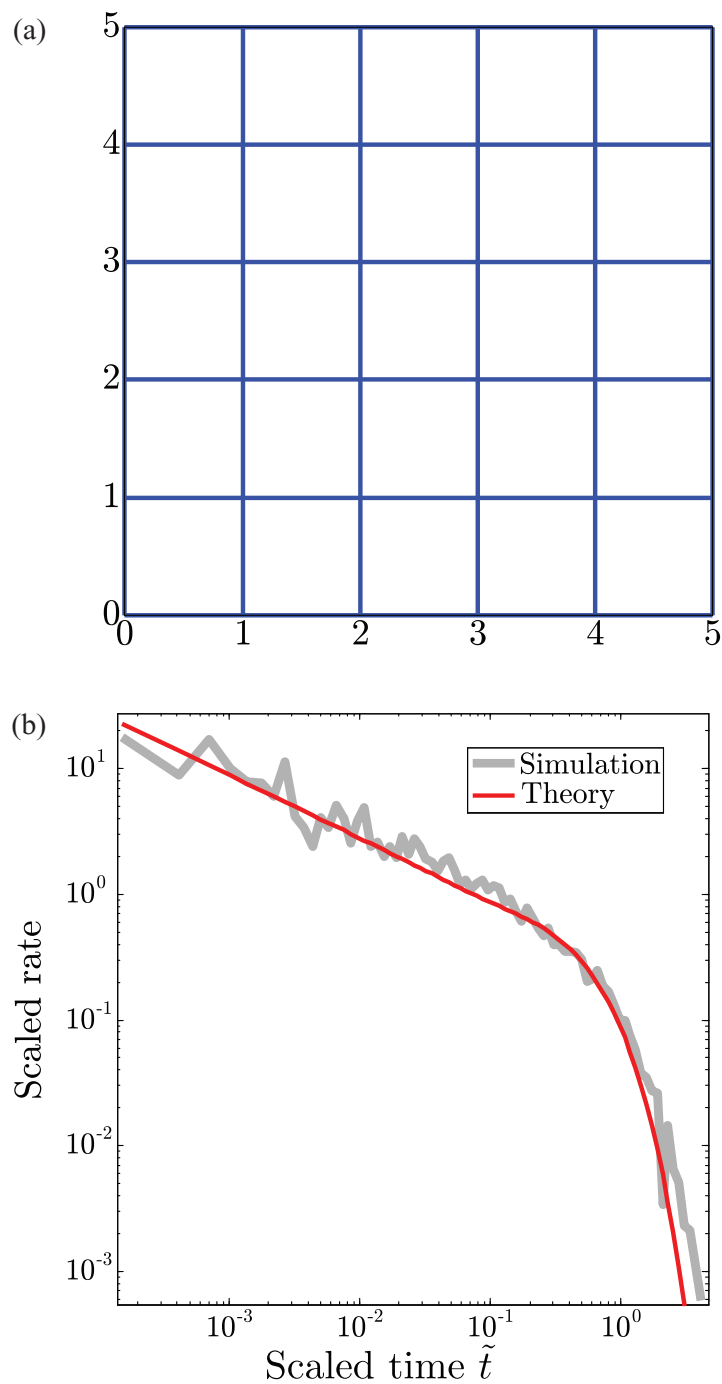

FIG. 3. Validation of our Brownian motion code. To construct the scaled rate vs time plot, we fit the parallel plate solution (29) to the probability density function for arrival time, which we get from the simulation. The fitting procedure yields a timescale $\tau$ and a mass scale $\mathcal{M}$, which we then use to scale time and rate: scaled time $\tilde{t}$ is defined by $t / \tau$; scaled rate, by $\dot{Q} /(\mathcal{M} / \tau)$. (a) Validation geometry, a fractured (blue) $5 \times 5$ lattice. (b) Simulation (jagged6 line) matches the theory from the parallel plate model (smooth line).

emergence of this power law is the most striking feature of Fig. 4. It suggests that the rate vs time plot might contain information about the geometry of the underlying fracture network and in particular about the power-law distribution of fractures forming the network. The exponent here for the decline of gas production is $D=-1.7$.

To examine the relationship between the power-law decline and fracture network geometry, we compared production decline for numerous fracture networks with different fracture length exponents $e$ and/or filling fractions $p$. The fracture length exponent $e$ controls the structure of the fracture network, such that small values result in networks made up of a few long fractures and large values lead to networks with numerous short fractures. (In the limit of $e \rightarrow \infty$, one recovers the classic bond percolation problem in which the size of

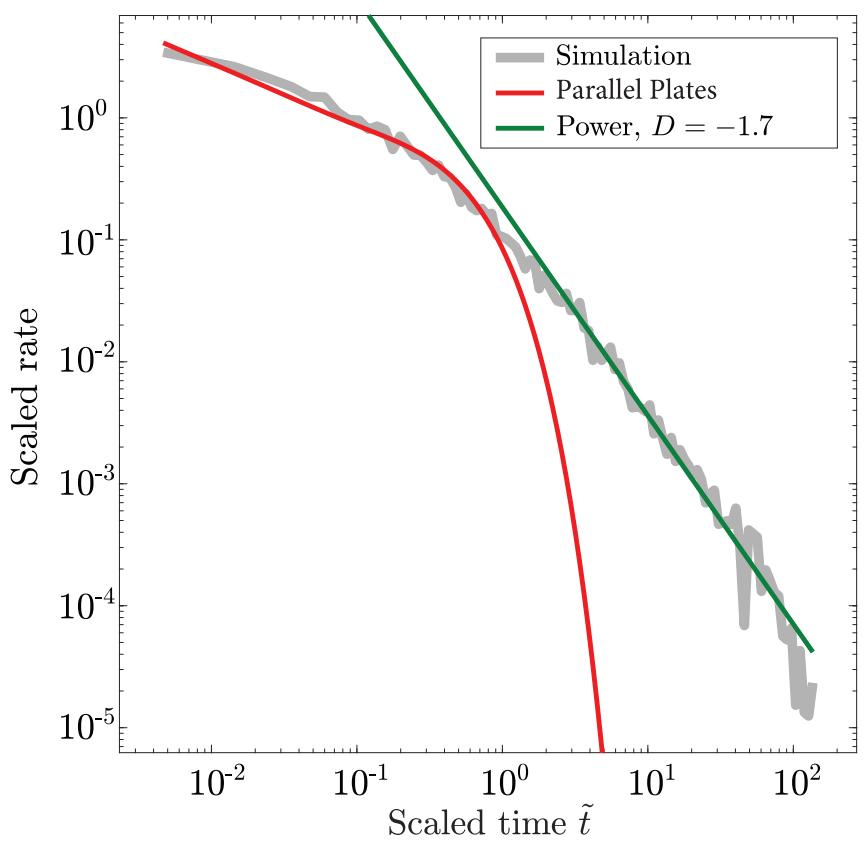

FIG. 4. Production decline for the fracture network in Fig. 2 (jagged line). The rate initially declines according to the solution for the parallel plate geometry, Eq. (29) (smooth curving line), but then transitions to a power-law decline (straight line).

every fracture is one lattice spacing.) The filling fraction $p$ controls the density of the fracture network.

As an example, Fig. 5 presents the geometry and rate for a network with $e=2$ and $p \approx 40 \%$. The exponent for the power decline here is $D=-1.4$, which is smaller in magnitude than the exponent for the network with $e=1.2$ and $p \approx 40 \%$.

Thus our Monte Carlo simulations suggested that the structure and density of fracture networks manifested themselves through a power-law decline in the time history of production rate. If the correspondence between fracture network geometry and production decline were one to one and could precisely be identified, we would be able to arrive at conclusions about fractal network geometry from the production decline. The concern with this method was that it was being applied in a relatively small, finite domain. We decided that limitations on precision and computational efficiency in the Monte Carlo method would make it worthwhile to develop an analytical approach capable of solving the problem exactly in an infinite external domain.

We developed such an approach and proceed to discuss it in detail. The methods were first described in [13], but the brevity of that discussion, as well as numerous technical improvements leading to a hundred-fold increase in solution speed justify a revised description, to which we now turn.

\section{EXACTLY SOLVABLE DISCRETE MODEL}

\section{A. Model definition}

We define an exactly solvable model on a square lattice. Every site of the lattice has a binary index associated with it and a continuous variable. The binary index determines whether the site is rock or an absorber. The continuous variable represents a density of gas. If the site is rock, then gas 
(a)
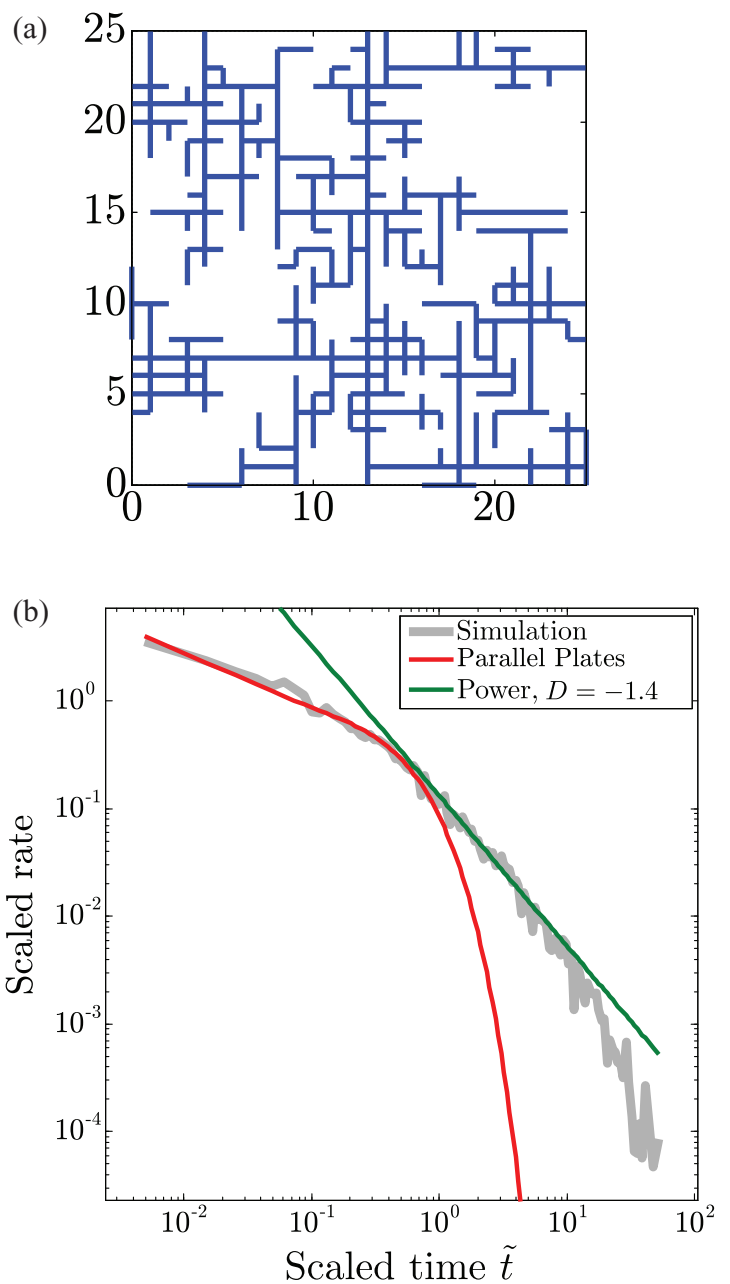

FIG. 5. Geometry and decline rate for structure with $e=2$ and $p \approx 40 \%$. (a) Fracture network with $e=2$ and $p \approx 40 \%$. (b) Production rate (jagged line) initially declines according to the solution for the parallel plate geometry (smooth curve), Eq. (29), but then transitions to a power-law decline (straight line).

diffuses through it as a conserved quantity. If the site is an absorber, any gas that arrives at it immediately disappears from the system. One can think of the absorbers as sites along a fracture network that conducts gas rapidly to the surface.

This model differs from the one in the previous section in a subtle way. In the previous section as well as here, the fracture network is defined on a square lattice. However, in the previous section the fractures were viewed as infinitely thin and gas density was defined everywhere in a two-dimensional plane. Here the gas density is defined only on the (countably infinite) sites of the lattice. Therefore the two models give different results at short times $t \leqslant a^{2} / \alpha=1$, where $a$ is the lattice spacing and $\alpha$ is the diffusivity. On long timescales, equivalently over large distance scales, the models are the same.

Sites of the square lattice are denoted by $\vec{R}_{j}$, or more simply just by $j$. Sites $j$ and $j^{\prime}$ on the lattice are connected by links of strength $k_{j j^{\prime}}=k_{j^{\prime} j}$. We restrict ourselves to nearest-neighbor coupling

$$
k_{j j^{\prime}}= \begin{cases}1 & \text { if } j \text { and } j^{\prime} \text { are nearest neighbors } \\ 0 & \text { otherwise. }\end{cases}
$$

Each site is either intact rock, or an absorber, which we indicate through a variable $\theta_{j}$ by

$$
\theta_{j}= \begin{cases}1 & \text { if } j \text { is rock, not an absorber } \\ 0 & \text { if } j \text { is an absorber. }\end{cases}
$$

On every site $j$ there lives at every time a density of gas $\rho_{j}(t)$. We denote the state vector of densities by $|\rho(t)\rangle$, and the density at site $j$ by

$$
\rho_{j}(t)=\langle j \mid \rho(t)\rangle .
$$

We use dimensionless variables, where distance is in units of a lattice spacing $a$, time is in units of $a^{2} / \alpha$, and $\alpha$ is the diffusivity of gas. The time evolution of $\rho$ is given by

$$
\dot{\rho}_{j}(t)=\sum_{j^{\prime}} \theta_{j} k_{j j^{\prime}}\left[\rho_{j^{\prime}}(t) \theta_{j^{\prime}}-\rho_{j}(t)\right] .
$$

Whenever a site $j$ is an absorber $\left(\theta_{j}=0\right)$, the density of gas located there does not change. At a rock site $j\left(\theta_{j}=1\right)$ that has as neighbor $j^{\prime}$ an absorber $\left(\theta_{j^{\prime}}=0\right)$, gas flows from $j$ to $j^{\prime}$ as if the density at $j^{\prime}$ is 0 . Since the density at $j^{\prime}$ does not change, the gas that flows in this way exits the system; conceptually it is transported to the surface out of the rock. The time evolution can be written more compactly as

$$
|\dot{\rho}(t)\rangle=\hat{H}|\rho(t)\rangle
$$

where

$$
\left\langle j|\hat{H}| j^{\prime}\right\rangle=H_{j j^{\prime}}=\theta_{j}\left[k_{j j^{\prime}}-B \delta_{j j^{\prime}}\right] \theta_{j^{\prime}} ; \quad B \equiv \sum_{j^{\prime}} k_{j j^{\prime}}=4
$$

We choose an initial density given by

$$
|\rho(0)\rangle=\left|\rho_{\mathrm{i}}\right\rangle, \quad\left\langle j \mid \rho_{\mathrm{i}}\right\rangle=\rho_{j}(0)=1 .
$$

We want to know the total amount of gas $Q$ that has exited from the lattice by time $t$. This is given by summing over $j$ :

$$
Q(t)=\sum_{j}\left[\rho_{j}(0)-\rho_{j}(t)\right]=\left\langle\rho_{\mathrm{i}} \mid \rho_{\mathrm{i}}\right\rangle-\left\langle\rho_{\mathrm{i}} \mid \rho(t)\right\rangle,
$$

where $\left\langle\rho_{\mathrm{i}}\right|$ is the transpose of $\left|\rho_{\mathrm{i}}\right\rangle$.

Because Eq. (6) is an infinite set of coupled equations, it is impossible to solve directly by any elementary means. We divide the solution into two phases. In most of our example problems, the system has one or more enclosed interior regions, all finite, and then an exterior region which is infinite. Intuitively it is clear that the solutions of disconnected interior regions are completely independent from each other, and independent of the solution of the exterior problem. Therefore, we solved these problems separately.

To do so, we modified the time evolution matrix of Eq. (8) twice, first to address the interior problems, and second to address the exterior problem. The modification for the interior problems is straightforward, since it is accomplished by limiting the equation of motion to a subspace. To address the exterior problem, the equation of motion has to be thought through in a new way. The solution of the problem once it is recast is so technically elaborate that we relegate most of 


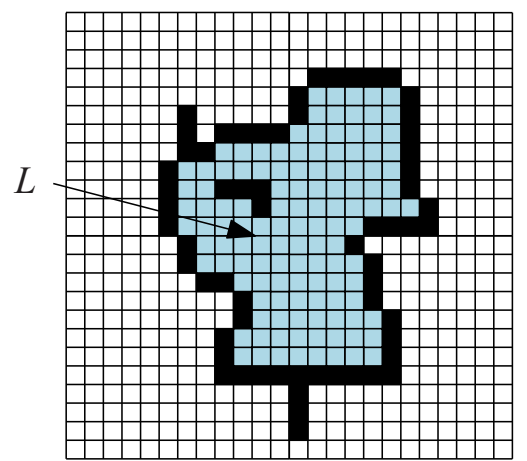

FIG. 6. Illustration of the interior of a fractured network. The absorbers are the black squares, and these are indicated by the condition $\theta_{j}=0$. The space $L$ is the space of all sites interior to the absorbers but not absorbers themselves: light blue (or gray), indexed by $l$.

it to a set of Appendixes. The remainder of this section is devoted to the two sets of solutions and to presentations of test problems as a prelude to applying the solutions to address physical questions.

To assist presentation of the two modified time evolution problems, we illustrate various subsets of lattice sites in Figs. 6 and 7.

\section{B. Solution for enclosed interior region}

Consider a region of the two-dimensional lattice surrounded by absorbers, and focus only on gas absorption in this interior region (Fig. 6). Project down onto the finitedimensional space $L$ of sites $j$ where $\theta_{j}=1$, (Fig. 6). Index this space with $l$. Then the solution of the interior problem is given by Eq. (7) with

$$
\left\langle l|\hat{H}| l^{\prime}\right\rangle=H_{l l^{\prime}}=k_{l l^{\prime}}-B \delta_{l l^{\prime}} .
$$

The matrix $\left\langle l|\hat{H}| l^{\prime}\right\rangle$ has $-B$ down the diagonal, and every row and every column has between zero and four off-diagonal entries equal to 1 . Any row or column with fewer than four

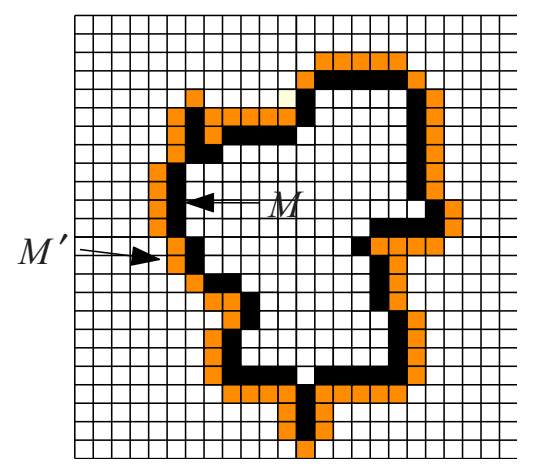

FIG. 7. Illustration of the exterior of a fractured network. Space $M$ is the set of all absorbers in contact with the exterior: black squares, indexed by $m$. Space $M^{\prime}$ is the set of all nearest neighbors of absorbers that are not themselves absorbers and in contact with the exterior: orange (or gray), indexed by $m^{\prime}$. Space $N$ is the union of $M$ and $M^{\prime}$ : black and orange (or gray) squares, indexed by $\nu$. Space $O$ is the set of absorbers in contact with the exterior and all exterior sites: black squares plus everything outside them. nonzero off-diagonal entries represents a lattice site with one or more neighboring absorbers. The factors $\theta_{j}$ from Eq. (8) are gone because only sites where $\theta_{j}=1$ are left in the system. In this space $L, \hat{H}$ has a complete set of eigenvectors $|\alpha\rangle$ so that

$$
1=\sum_{\alpha}|\alpha\rangle\langle\alpha| \quad \text { and } \hat{H}|\alpha\rangle=E_{\alpha}|\alpha\rangle .
$$

One can write the solution of Eq. (7) as

$$
|\rho(t)\rangle=\sum_{\alpha} e^{E_{\alpha} t}|\alpha\rangle\left\langle\alpha \mid \rho_{\mathrm{i}}\right\rangle .
$$

Clearly all the eigenvalues $E_{\alpha}$ must be negative, or else there would exist initial conditions for which the amount of gas in the lattice increases, and that is impossible. At time $t$ the amount of gas that has been produced is

$$
Q(t)=\left\langle\rho_{\mathrm{i}} \mid \rho_{\mathrm{i}}\right\rangle-\sum_{\alpha} e^{E_{\alpha} t}\left|\left\langle\alpha \mid \rho_{\mathrm{i}}\right\rangle\right|^{2},
$$

and the production rate is (note again that all eigenvalues are negative)

$$
\dot{Q}=-\sum_{\alpha} E_{\alpha} e^{E_{\alpha} t}\left|\left\langle\alpha \mid \rho_{\mathrm{i}}\right\rangle\right|^{2} .
$$

These expressions are practical so long as finding all the eigenvalues is practical. By way of reference, for a $100 \times 100$ two-dimensional lattice, such as the interior region depicted in Fig. 2(b), for which the matrix $\hat{H}$ is on the order of $10000 \times$ 10000 , standard eigenvalue solvers can find all eigenvalues and eigenvectors in a few seconds on a desktop computer. The matrix is sparse, but for a problem of the scale just mentioned, the sparse matrix eigenvalue solvers are much slower, less accurate, and find only a subset of the eigenvalues rather than all of them as we require. For the largest systems considered in this paper (Sec. IV F) the interior region was partitioned into disconnected subregions, the eigenvalue problem was solved in each of them, and the results summed at the end to give total interior production. Finding all the disconnected interior regions is an easy numerical problem since one simply needs to place a boundary around all the absorbers and then run through all the sites inside this boundary, checking for regions connected to each other.

We did not pursue the formal inverse mathematical problem of deducing the fracture network from the production curves. Based on the physically motivated analyses that follow it seems certain that the mathematical problem is ill-posed, for variations in the initial conditions are amplified exponentially by the diffusion operator, Eq. (6), when time is reversed.

\section{Solution for infinite system}

Any collection of absorbers in contact with an infinite twodimensional lattice must have some absorbers that draw on an unbounded amount of gas. While formally one can still employ Eq. (13), the number of eigenvalues and eigenvectors is infinite, and a direct approach to diagonalizing the diffusion operator fails. This requires a completely different and more elaborate formal approach.

While the formalism is necessary to achieve our aims, the main purpose of this paper is to employ this formalism to answer questions about collection networks. Therefore we 
develop the formalism in a series of Appendixes. Here we only describe the purpose of each Appendix.

Appendix A Obtain an equation of motion that is identical to Eq. (6) in the exterior region $O$ (Fig. 7) but keeps all gas in the interior regions from ever changing if the interior begins with uniform density $\rho_{0}$.

Appendix $B$ Present expressions for lattice Green's functions in the infinite square lattice and find methods for the computation of the matrix elements for large distances. This task does not appear to have been achieved previously, but is necessary for the ensuing results.

Appendix $C$ Use a $T$ matrix formalism to reduce the solution of the infinite exterior problem to a finite matrix problem defined on the domain $N$ (Fig. 7).

Appendix $D$ Compute gas production $\dot{Q}$ from the solution of the finite matrix problem.

We summarize the net result of the whole procedure. In the Appendixes we use standard bra and ket notation from the Green's function literature, while here we repeat key expressions in matrix form. Let $H^{0}$ be the matrix corresponding to a diffusion problem on an infinite square lattice without absorbers,

$$
H_{j j^{\prime}}^{0}=k_{j j^{\prime}}-4 \delta_{j j^{\prime}}
$$

and let $G^{0}(E)$ be a matrix that solves the equation, for $E$ in the complex plane, $G^{0}(E)\left(E-H^{0}\right)=1$. Define a matrix $H^{1}$ on the space $N$ with the following matrix elements: consider a site $m$ that is an absorber bordering the exterior (space $M$ in Fig. 7), and suppose it has $n_{m}$ neighboring exterior rock sites. Then $H^{1}$ has diagonal elements

$$
H_{m m}^{1}=n_{m}
$$

The off-diagonal elements, for each $m^{\prime}$ that is a neighboring exterior rock site of $m$ (space $M^{\prime}$ in Fig. 7), are

$$
H_{m m^{\prime}}^{1}=H_{m^{\prime} m}^{1}=-1 \text {. }
$$

Define the vector $\overrightarrow{\mathcal{H}}$ with components

$$
\mathcal{H}_{v} \equiv \sum_{v^{\prime}} H_{v v^{\prime}}^{1}
$$

Here $v$ lives in space $N$ of Fig. 7. Then find the vector $\overrightarrow{\mathcal{T}}(E)$ that solves the following matrix equation

$$
\left[1-H^{1} G^{0}(E)\right] \overrightarrow{\mathcal{T}}(E)=\overrightarrow{\mathcal{H}} .
$$

This matrix equation is finite dimensional, since the matrix $H^{1}$ and the vectors $\overrightarrow{\mathcal{T}}(E)$ and $\overrightarrow{\mathcal{H}}$ have indices that run over the finite space $N$. The matrix $G^{0}$ is defined on the infinite square lattice, but here one uses only the matrix elements $G_{v v^{\prime}}^{0}(E)$ for $v$ and $v^{\prime}$ in space $N$.

Then the gas production rate is given by an integral over the argument $E$ of $\overrightarrow{\mathcal{T}}(E)$ through

$$
\dot{Q}=-\int_{-8}^{0} \frac{d \lambda}{\pi \lambda} e^{\lambda t} \operatorname{Im} \sum_{v} \mathcal{T}_{v}(\lambda) .
$$

\section{Solutions of test problems}

\section{One absorber}

The simplest possible problem that makes use of this formalism is a single absorber in an infinite square lattice. $|\mathcal{T}\rangle$ has five components. The first corresponds to the absorber at $t=0$, and the remaining five correspond to the four neighbors of the absorber. Only $\mathcal{T}_{0}$ has a nonzero imaginary part; the remaining values are $\mathcal{T}_{v}=-1$, where $v=1, \ldots, 4$,

$$
\mathcal{T}_{0}(\lambda)=4 \frac{G_{10}(\lambda)}{G_{00}(\lambda)}=\lambda+4-\frac{G_{00}^{*}(\lambda)}{\left|G_{00}(\lambda)\right|^{2}},
$$

and $G_{00}^{*}(\lambda)$ is the complex conjugate of $G_{00}(\lambda)$. So

$$
\operatorname{Im} \mathcal{T}_{0}(\lambda)=-\operatorname{Im} \frac{1}{G_{00}(\lambda)}
$$

This is the only contributor to gas production, as shown in Eq. (D5). As in Eq. (D7), define

$G_{00}^{l}(f)=G_{00}\left(-e^{-f}\right) ; \quad \mathscr{T}(f) \equiv \lim _{\eta \rightarrow 0} \operatorname{Im} \sum_{\nu} \mathcal{T}_{v}\left(-e^{-f}-i \eta\right)$.

We find the spectrum and production rate shown in Fig. 8:

$$
\dot{Q}=\int_{-\ln 8}^{\infty} \frac{d f}{\pi} e^{-t e^{-f}} \mathscr{T}(f)=\int_{-\ln 8}^{\infty} \frac{d f}{\pi} e^{-t e^{-f}} \operatorname{Im} \frac{-1}{G_{00}^{l}(f)} .
$$

For the integral to converge, one has to take values of $f$ up to around 2000, which correspond to values of $\lambda$ on the order of $e^{-2000}$. Asymptotic values of the lattice Green's function are available [21]. In the limit as $\lambda \rightarrow 0^{-}$,

$$
\lim _{\lambda \rightarrow 0^{-}} \operatorname{Im} G_{00}=\frac{1}{4}, \quad \operatorname{Re} G_{00}(\lambda)=\frac{1}{4 \pi} \ln \left(-\frac{32}{\lambda}\right) .
$$

Therefore, for $f<300$, we compute $G_{00}^{l}(f)$ directly, while for $f>300$ we use the asymptotic expression

$$
\mathscr{T}(f) \approx \frac{1}{1 / 4+1 / 4 \pi^{2}[f-\ln (1 / 32)]^{2}} .
$$

An asymptotic procedure of this sort works in the general case, as we discuss below. That is, for large enough $f$, every finite collection of absorbers leads to an integrand of this form, but with different values in place of $1 / 32$.

A good way to check whether the procedure has been carried out correctly is to check a sum rule that applies when $t=0$. The rate of gas production at $t=0$ always equals the number of external nearest neighbors of the sites belonging to $N$. The reason for this is that at time $t=0$ the gas density is uniform and equals 1 everywhere, and it flows into every absorber at rate 1 . In this particular case, the sum rule is obeyed and takes the form

$$
4=\dot{Q}(0)=\int_{-\ln 8}^{\infty} \frac{d f}{\pi} \operatorname{Im} \frac{-1}{G_{00}^{l}(f)} .
$$

Given the definition of $G_{00}(\lambda)$ in terms of elliptic function Eq. (B14), this is not at all self-evident and could not be obeyed unless both the theoretical results and numerical implementation are correct. 


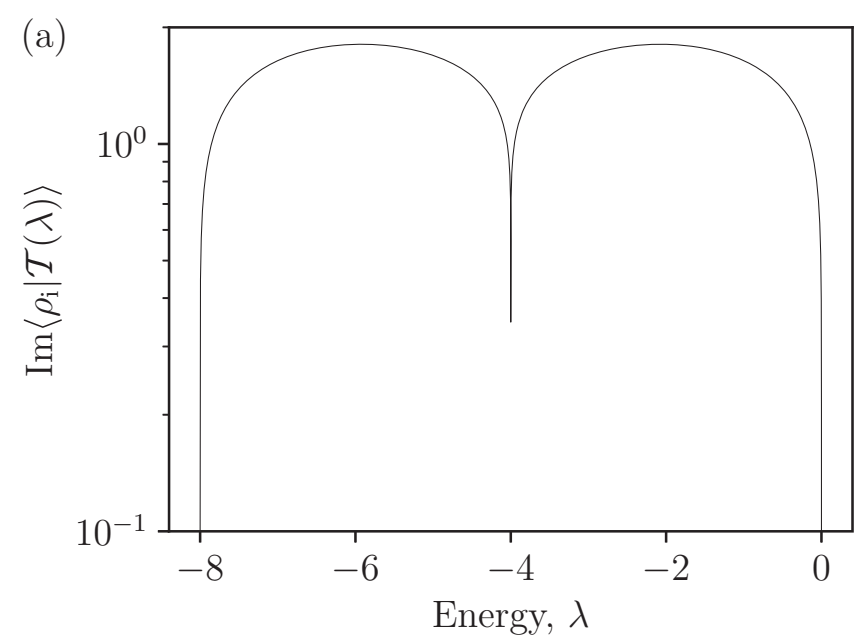

(b)

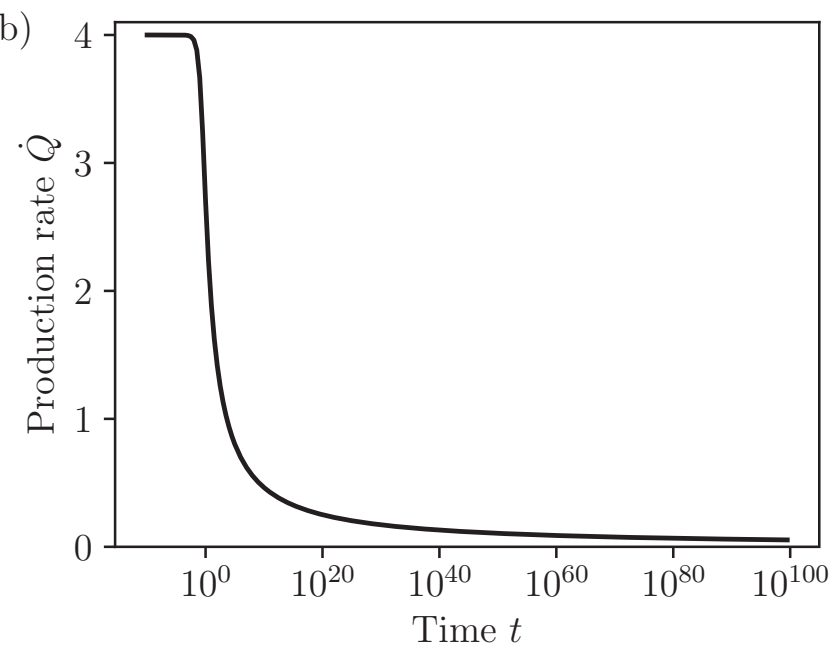

FIG. 8. Spectrum and production for a single absorber. (a) Continuous spectrum on the negative real axis for single absorber. (b) Gas production, single absorber.

\section{Square}

We now turn to a case whose solution we will employ repeatedly in what follows. It is a square shown in Fig. 9(a). The interior problem for region $L$ (Fig. 6) is of size $64^{2} \times 64^{2}$. The sum rule for initial production from the interior gives $\dot{Q}(0)=256=4 \times 64$, which serves as check on the computations. Gas production from the interior region comes from Eq. (15), and the result appears in Fig. 9(b).

We can compare this with an analytical solution. It is the solution $\dot{Q}_{\text {plates }}(t)$, which is the rate of diffusive flow to two absorbing plates separated by distance 2 [6]:

$$
\dot{Q}_{\text {plates }}(t)=2 \sum_{n=0}^{\infty} e^{-(2 n+1)^{2} \pi^{2} t / 4} .
$$

The comparison of this solution with the solution for the square is performed in the following way. The flow rate for the interior of the square is

$$
\dot{\mathcal{Q}}_{\text {int }}(t)=\frac{\mathcal{V}}{\tau} \dot{Q}_{\text {plates }}(t / \tau)
$$

(a)
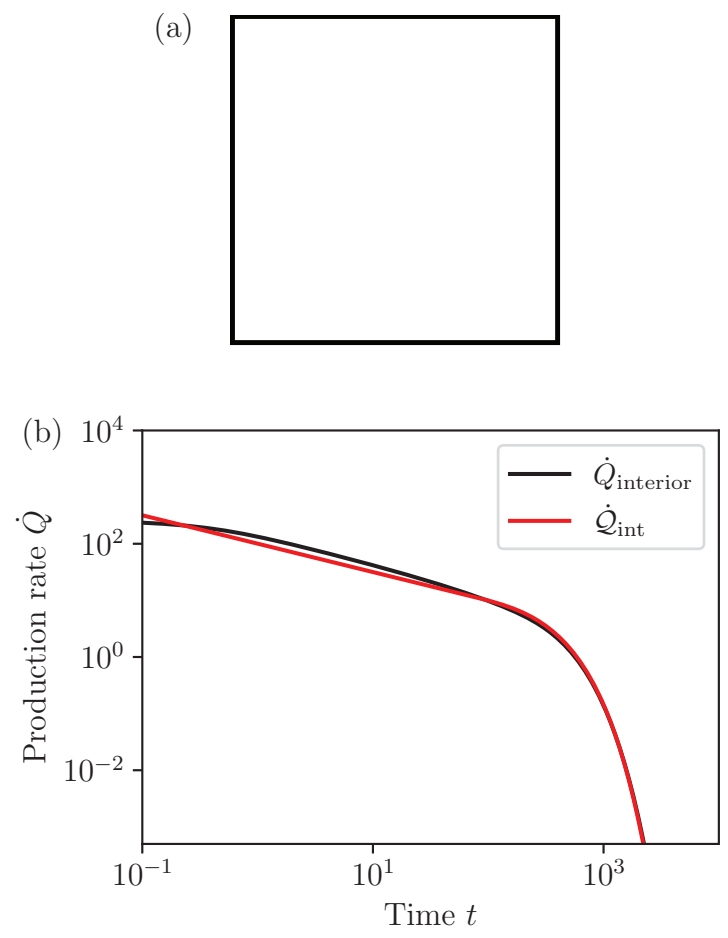

FIG. 9. (a) Square with interior of size $64 \times 64$. (b) Interior production from the square, comparing exact computation $\dot{Q}_{\text {interior }}$, Eq. (15), with approximate analytical expression $\mathcal{Q}_{\text {int }}$, Eq. (29), scaled as described in the text.

The two-dimensional interior volume $\mathcal{V}$ of the region is $64^{2}$. The interference time for parallel plates separated by distance $2 d$ in a medium of diffusivity $\alpha$ is $d^{2} / \alpha$ [6]. For our square with unit diffusivity we have instead $\tau=\frac{1}{2}(64 / 2)^{2}$. The leading factor of $1 / 2$ is due to the fact that there is twice the surface area at which gas can be collected as in for the parallel plates, so one should expect the interference time to be cut in half. This is indeed an exact result in the continuum limit, and one sees from Fig. 9(b) that the solution of the discrete lattice model agrees with it well apart from small deviations at early times.

We now turn to solution of the exterior problem for the square. When employing Eq. (D8) we find that our procedures to compute the integrand are stable up to $f \approx 2000$ and become unstable and inaccurate for larger values. Therefore it is advantageous to see if the function $\mathscr{T}(f)$ defined in Eq. (D7) can adequately be represented by an asymptotic expression for large $f$. The form suggested by Eq. (26) is

$$
\frac{1}{\mathscr{T}(f)} \approx \mathscr{T}_{1}+\mathscr{T}_{2}\left(f-f_{0}\right)^{2} \quad \text { as } f \rightarrow \infty .
$$

In the range $f \in[1200,3000]$, this asymptotic expression reproduces direct calculation of $\mathscr{T}(f)$ up to nine decimal places. The coefficients $\mathscr{T}_{1}$ and $\mathscr{T}_{2}$ that enable this fit are identical to their values in Eq. (26). Indeed, we find they never vary for any structure we have examined. It seems clear this could be proved, but we have not sought the proof. We replace $f_{0}$ with $\ln \left(\sigma / \sigma_{0}\right)$-we will choose $\sigma_{0}$ to give $\sigma$ geometrical 


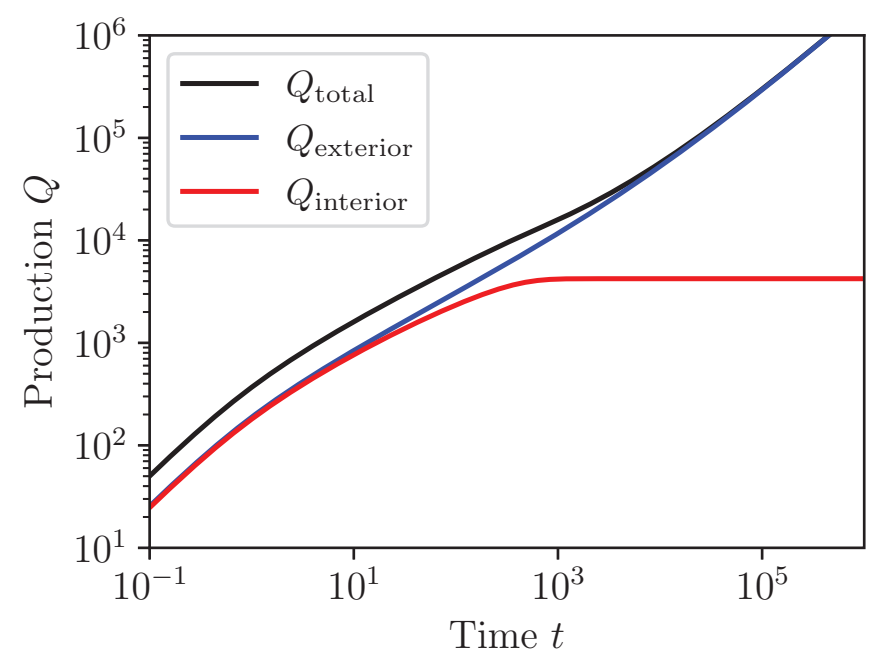

FIG. 10. Cumulative production for flow to network shown in Fig. 9(a). From top to bottom, total, exterior, and interior production.

meaning - and write

$$
\begin{aligned}
\dot{Q}= & \frac{1}{\pi} \int_{-\ln 8}^{g} d f \exp \left(-t e^{-f}\right) \mathscr{T}(f) \\
& +\frac{1}{\pi} \int_{g}^{\infty} d f \frac{\exp \left(-t e^{-f}\right)}{1 / 4+1 /\left[4 \pi^{2}\right)\left(f-\ln \left(\sigma / \sigma_{0}\right)\right]^{2}}
\end{aligned}
$$

For most cases we studied, the separation parameter $g=300$ is large enough, although for the structure in Sec. IV F it needed to be raised to 1000, as indicated by residuals of the fit in Eq. (31). In the current instance we find $\sigma / \sigma_{0}=1205$, and define $\sigma_{0}$ - for all fracture networks - as

$$
\sigma_{0}=3.506 \text {. }
$$

We will allow $\sigma$ to vary from structure to structure, and in the current case we find

$$
\sigma=4225=65^{2}
$$

The reason for this definition looks forward to scaling properties of $\dot{Q}(t)$ and $Q(t)$ which we will discuss in Sec. IV A. We choose $\sigma_{0}$ so that $\sigma$ is identical to the exterior volume $\mathcal{V}_{\text {ext }}=65^{2}$ of the fracture network. We will show that since for this one structure $\sigma$ equals the exterior volume, $\sigma$ obtained using Eq. (31) for other squares will give their volumes to high precision. For more complicated structures, this procedure gives a definition of the exterior volume, a constant that determines the universal form of their production at late times (Sec. IV C).

The exterior problem produces a matrix for Eq. (20) of size $532 \times 532$. Initial production from the exterior is $\dot{Q}(0)=$ 260 , and we recover this value from Eq. (31). This sum rule could not be obeyed if the asymptotic approximation in Eq. (30) were not accurate. The sum of interior and exterior production rates and cumulative production from 0.1 to $10^{6}$ time units appear in Figs. 9(b) and 10. Note that for times up to around 10 the interior and exterior production are nearly identical. This makes sense because the fracture network is a thin closed circuit, and the inside and outside of it are locally the same except at the corners.

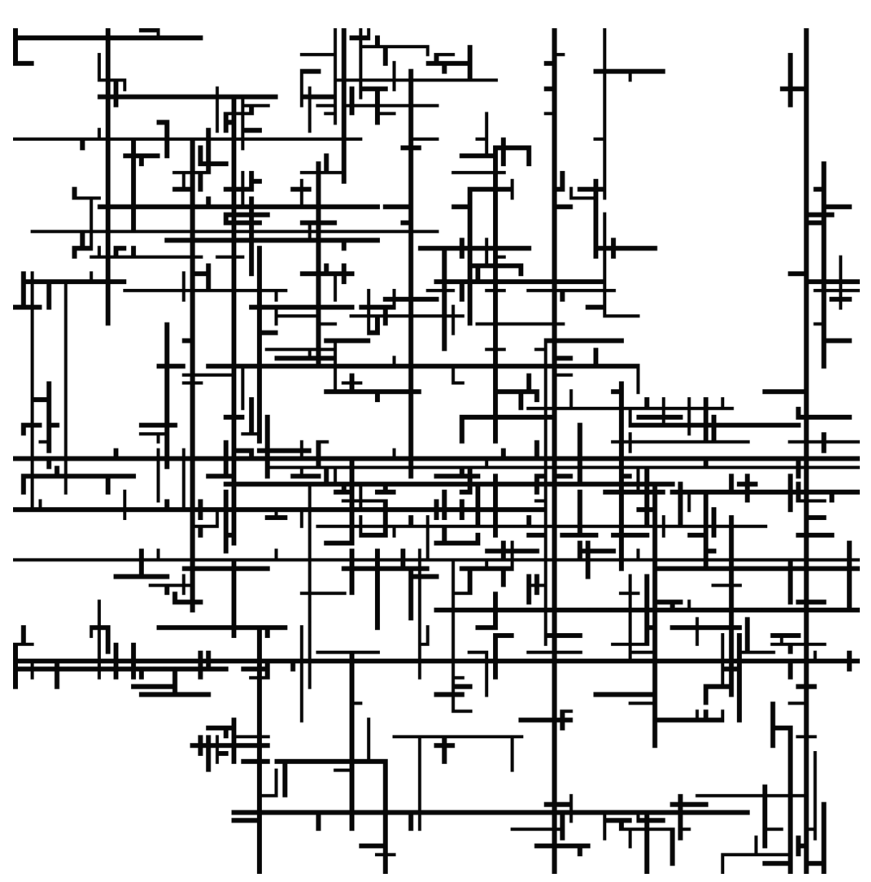

FIG. 11. Network of absorbers used as example.

\section{Fractal fracture network}

As a final test case, consider a network generated from Eq. (1) with $e=1.2$ and $p=25 \%$ and shown in Fig. 11. The interior space $L$ has 3080 sites, and therefore the interior solution has 3080 eigenvalues and eigenvectors in Eq. (15). The sum rule for the interior gives $\dot{Q}(0)=3264$. There are more neighbors of absorbers than absorbers because each absorber can have as many as four neighbors. The production rate and cumulative production from the interior of this network are depicted in Fig. 13 below. The interior production was found by finding all the 195 disconnected closed regions inside the network, ranging in size from 1 site to 1417 sites, and applying Eq. (15) to each of them in turn. The process of finding all the disconnected regions is numerically quite fast as the algorithm is linear in the overall size of the structure.

Computation of the external flow to this network was at first challenging. It was in the attempt to calculate production for this structure that we developed the integration contour of Fig. 23 in Appendix D. If one tries to compute the integral of Eq. (31) by integrating along the negative real axis, it means integrating the function shown in Fig. 12(a). There are hundreds of peaks of width on the order of $10^{-3}$ and heights up to $10^{5}$. By contrast, integrating along the contour shown in Fig. 23 produces the integrand of Fig. 12(b). This is relatively smooth, has no peaks, and is fast to integrate. The sum rule $\dot{Q}(0)=4820$ is satisfied to seven decimal places at $t=0$, and this provides assurance that the computation is technically correct. For the asymptotic expression of Eq. (30), $\sigma=2419$. Despite geometrical complexity of the network, and complexity of the solution in the complex plane seen in Fig. 12(a), the solutions shown in Fig. 13 are somewhat anticlimactic in their smooth behavior. 

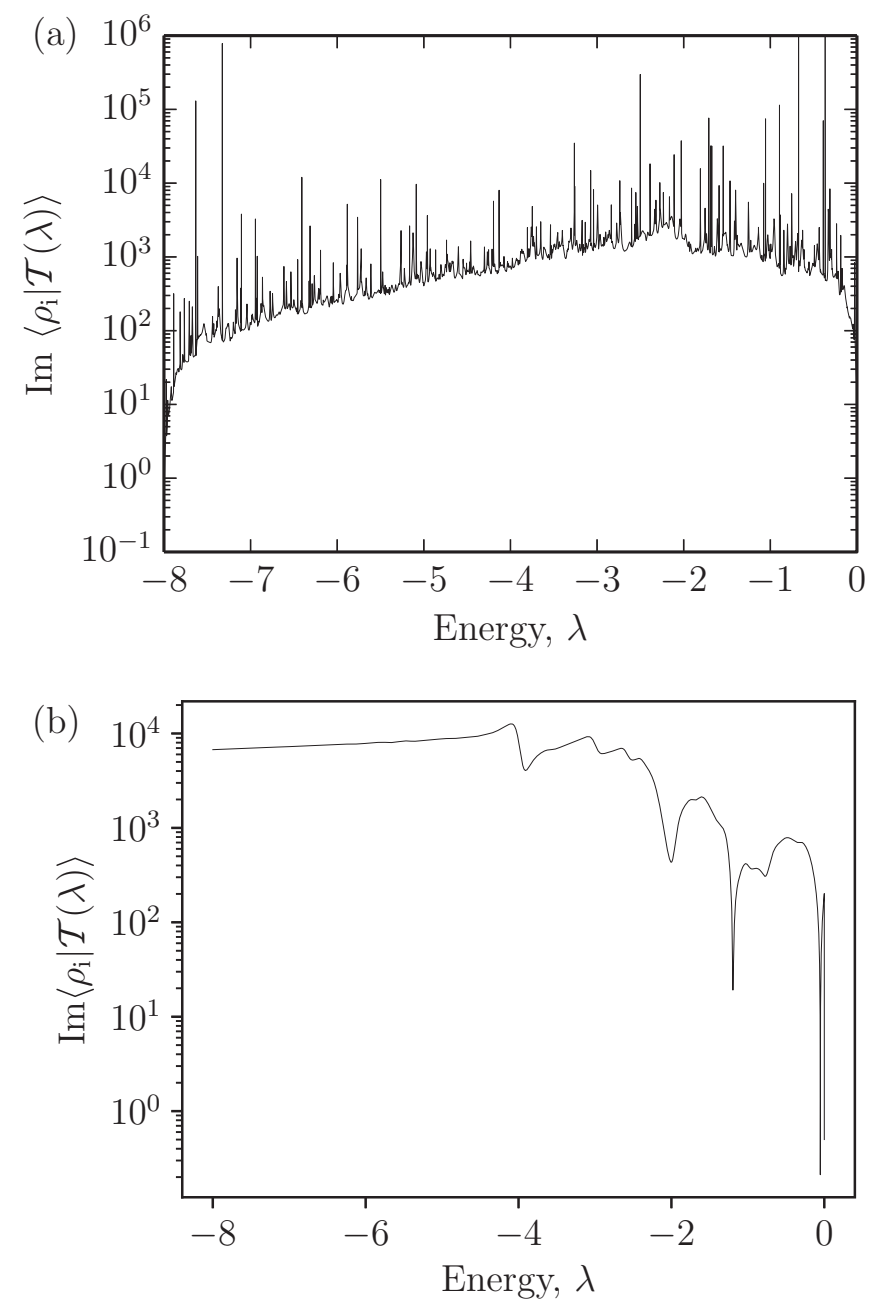

FIG. 12. Continuous spectrum from network of Fig. 11. (a) Continuous spectrum along negative real axis for network in Fig. 11. (b) Real part of continuous spectrum along contour in complex plane in Fig. 23, as function of real part of contour variable.

\section{APPLICATIONS AND INTERPRETATION OF EXACT SOLUTIONS}

\section{A. Dimensional form and scaling}

We now apply this formalism to settle questions about production from fracture networks.

To find scaling laws to relate solutions to each other, we put our results in dimensional form. Suppose that unit lattice spacing corresponds to the physical distance $a$, that unit dimensionless time corresponds to the time interval $t_{0}$, that dimensionless gas density corresponds to mass density $\rho_{0}$ and that the diffusivity is $\alpha$. Let $\tilde{Q}(\tilde{t})$ give dimensional production versus dimensional time where the unit of production is mass, and time $\tilde{t}=t \times t_{0}$ also carries units. The evolution equation for gas is

$$
\frac{d}{d \tilde{t}}|\tilde{\rho}(\tilde{t})\rangle=\frac{a^{2}}{t_{0}} \frac{\hat{H}}{a^{2}}|\tilde{\rho}(\tilde{t})\rangle .
$$
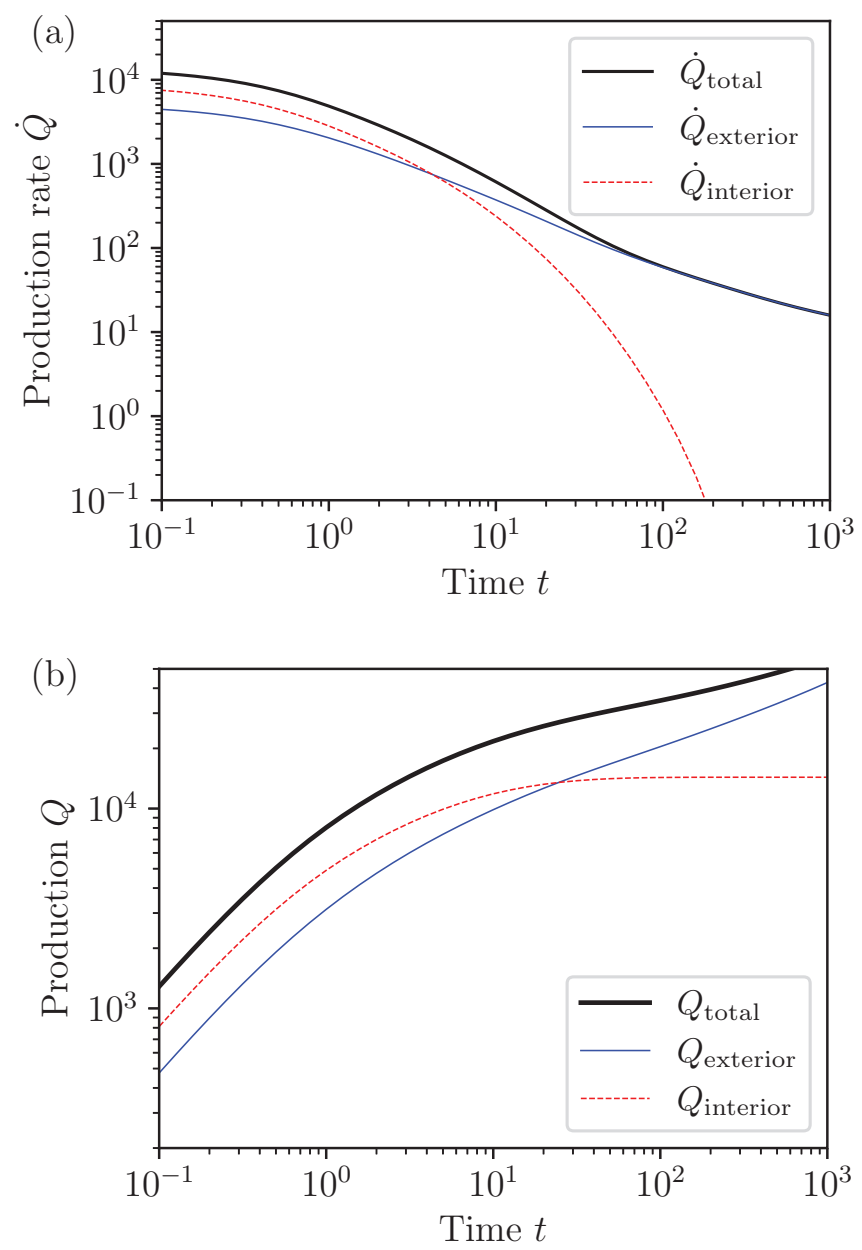

FIG. 13. (a) Gas production rate for network in Fig. 11. (b) $\mathrm{Cu}-$ mulative gas production from interior of network in Fig. 11.

In the continuum limit, $\hat{H} / a^{2}$ becomes the Laplacian operator $\nabla^{2}$. Therefore we identify the diffusivity as

$$
\alpha=\frac{a^{2}}{t_{0}} .
$$

We can generalize this expression. Suppose we refine the lattice spacing by a factor $s$, replacing $a$ by $a / s$, but that otherwise we are describing the same physical phenomenon and in particular keep $\alpha$ fixed. Let $\tilde{\rho}_{s}$ be the dimensional solution for the problem defined on the lattice refined by factor $s$. Then

$$
\frac{d}{d \tilde{t}}\left|\rho_{s}(\tilde{t})\right\rangle=\alpha s^{2} \frac{\hat{H}}{a^{2}}\left|\rho_{s}(\tilde{t})\right\rangle .
$$

Therefore $\tilde{\rho}_{s}(\tilde{t})$ describes the same problem in time as $\tilde{\rho}(\tilde{t})$ if one replaces $\tilde{t}$ by $\tilde{t} s^{2}$. Since the number of lattice squares corresponding to any particular physical region goes up by $s^{2}$, one has to divide the gas concentration $\rho_{o}$ by this value; that is,

$$
s^{-2} \tilde{\rho}_{s}\left(s^{2} \tilde{t}\right)=\tilde{\rho}(\tilde{t})
$$

We can now return to the dimensionless production function. We have found that if one refines the lattice by a factor $s$, 
the new solution $Q_{s}(t)$ obeys

$$
s^{-2} Q_{s}\left(s^{2} t\right)=Q(t) ; \Rightarrow \dot{Q}_{s}\left(s^{2} t\right)=\dot{Q}(t) .
$$

The expressions in Eqs. (37) and (38) are approximate at early times when lattice effects are important and become exact in the limit of late times when only large scales matter. We will use this scaling result frequently in what follows.

It may be helpful to mention typical values of a few variables $[6,14]$. The diffusivity $\alpha$ is on the order of $10^{-8} \mathrm{~m}^{2} / \mathrm{s}$. The spacing between fractures $d$ after well stimulation is around $1 \mathrm{~m}$. This means that the characteristic time to drain the interior of a structure is $10^{8} \mathrm{~s}$ or around three years.

\section{B. Apparent power law seen in Monte Carlo is not present in exact solutions}

We apply these scaling ideas as we return to examine the results of Sec. II again. From the Monte Carlo simulations it appears that gas production from a complex fractured network might reveal a signature of the network complexity in its late-time behavior through a power-law decay. It is also possible that the power law is an artifact of the finite region in which the simulations have been performed. We return to this question using the Green's function formalism of Sec. III.

Each of the numerical methods has its strengths and weaknesses. The kinetic Monte Carlo solutions have a stochastic element and can be performed only in a finite domain. The Green's function calculations are exact in an infinite domain, but they are performed on a lattice, and the behavior at short times is unrealistic. The extent of lattice effects depends on precisely how the lattice model is implemented. Figure 14 shows three separate realizations of the fracture network of Fig. 5. The fracture network was defined in a space of size $25 \times 25$. The first realization represents the network on a $25 \times 25$ lattice. The next realizations refine the network by factors $s$ of 2 and 6 respectively. Thus, in Fig. 14(c), there is a minimum of five empty lattice sites between the most closely spaced fractures.

We noted in the previous subsection that when a lattice is refined by scale factor $s$, the time needed for any diffusion process increases by the square of the scaling factor. We verify the scaling of Eq. (38) explicitly in Fig. 15(a). We want to reach the continuum limit, and rescaling by a factor of 6 is adequate to achieve this goal for times as little as $10^{-2}$, while the unscaled network shows lattice effects up to a time of 10. At late times production all comes from large distances, and only the rough external contour of the fracture network matters for production.

Now that lattice effects are under control, we compare the Green's function solution for the network on a lattice (scaled up by a factor of 6) with the kinetic Monte Carlo result. Figure 15 shows the comparison. We rescale time both for the Green's function solution and for the kinetic Monte Carlo solution so as to match the function $\mathcal{Q}_{\text {int }}$ from Eq. (29) at early times. The portion of the kinetic Monte Carlo solution we previously thought might describe a power law we now see results from the finite domain in which the solution was found. The Green's function solution, which is exact in an infinite domain, does not show a trace of such a power law, and we therefore conclude that fractal features of the fracture geome- (a)

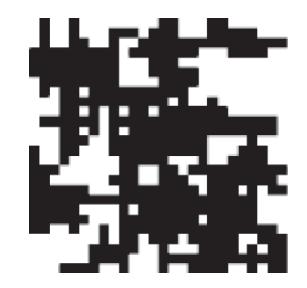

(b)

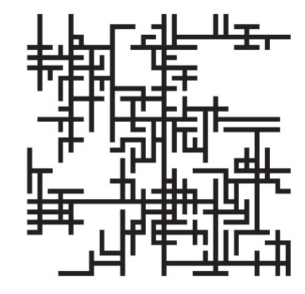

(c)

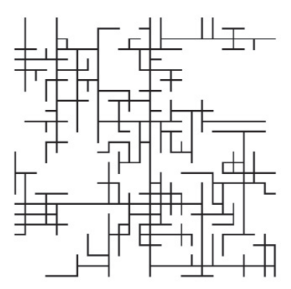

FIG. 14. Lattice realizations of the fracture network of Fig. 5 at three separate resolutions. (a) Fracture network of Fig. 5 on lattice whose spacing equals minimum distance between fractures. (b) Fracture network of Fig. 5 on lattice whose spacing equals half the minimum distance between fractures (c) Fracture network of Fig. 5 on lattice whose spacing equals one sixth the minimum distance between fractures.

try cannot be obtained from analysis of production decline in this way. In Sec. IV E we provide an analytical examination of the relation between power-law fracture networks and production power laws. We will show that the fractal structure of the collection network does reveal itself in production curves, but not in the way we originally thought.

\section{Very late-time behavior is universal}

In this section we show that at late times, all wells fall on a single production curve scaled by two variables. We return to Eq. (31) and write

$$
\dot{Q} \approx \frac{1}{\pi} \int_{-\infty}^{\infty} d f \frac{\exp \left(-t e^{-f}\right)}{1 / 4+1 /\left(4 \pi^{2}\right)\left[f-\ln \left(\sigma / \sigma_{0}\right)\right]^{2}} .
$$

This motivates the definition, sending $f \rightarrow f+\ln \left(\sigma / \sigma_{0}\right)$,

$$
\dot{Q}_{\mathrm{lt}}=4 \pi \int_{-\infty}^{\infty} d f \frac{\exp \left[-\left(t \sigma_{0} / \sigma\right) e^{-f}\right]}{f^{2}+\pi^{2}} .
$$

There is a more transparent but less accurate expression at very late times (vlt) given by

$$
\dot{Q}_{\mathrm{vlt}} \approx \frac{4 \pi}{\ln \left(t \sigma_{0} / \sigma\right)} \quad \text { and } \quad Q_{\mathrm{vlt}} \approx \frac{4 \pi t}{\ln \left(t \sigma_{0} / \sigma\right)} .
$$

What one sees from Eq. (39) is that the late-time behavior of $\dot{Q}$ depends on the single parameter $\sigma$, which sets a timescale. This late-time behavior describes production of all structures, including structures of different sizes. As we 

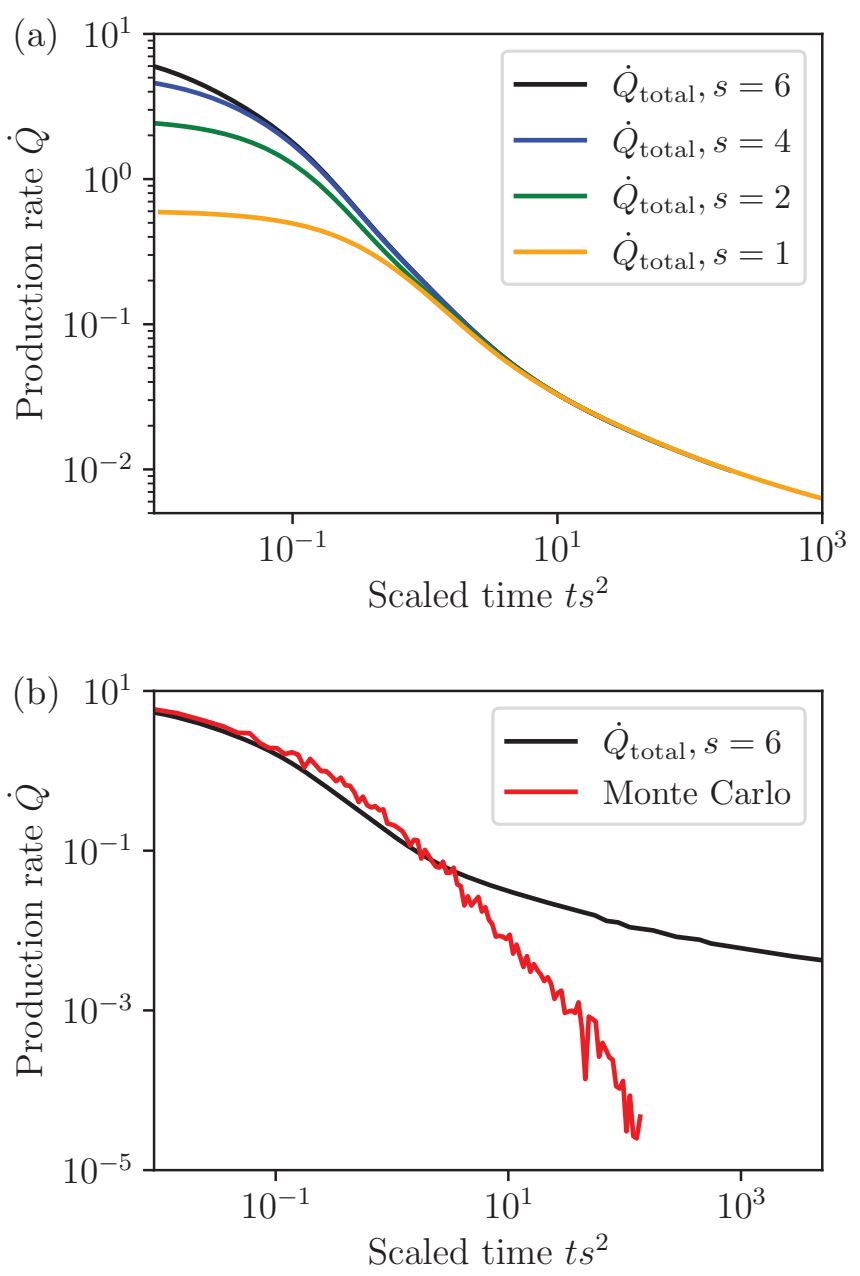

FIG. 15. Comparison of kinetic Monte Carlo and lattice Green's function result for fracture network of Fig. 5. (a) Convergence of Green's function solution as lattice of Fig. 5 is scaled by factors $s$ (from lowest curve to highest curve) of $1,2,4$, and 6 , and thus approaching a continuum limit where the early-time behavior is a $t^{-1 / 2}$ power law. The scaled time is time divided by the scaling factor squared, leading the curves to lie on top of one another. (b) Comparison of Green's function solution scaled by factor of 6 with kinetic Monte Carlo solution in Fig. 5(b).

showed in Sec. IV A, the late-time cumulative production of two structures must be unchanged when distances are rescaled by a factor $s$ and time is rescaled by $s^{2}$. How is this compatible with the dependence of Eq. (39) on a single parameter?

To answer this question, we examine cumulative production at late times, $Q_{\mathrm{lt}}$, which is given by

$$
\begin{aligned}
Q_{\mathrm{lt}}(t, \sigma) & =4 \pi \int_{-\infty}^{\infty} d f \int_{0}^{t} d t^{\prime} \frac{\exp \left[-\left(t^{\prime} \sigma_{0} / \sigma\right) e^{-f}\right]}{f^{2}+\pi^{2}} \\
& =4 \pi \sigma \int_{-\infty}^{\infty} d f \int_{0}^{t / \sigma} d t^{\prime} \frac{\exp \left[-\left(t^{\prime} \sigma_{0}\right) e^{-f}\right]}{f^{2}+\pi^{2}} \\
& =\sigma Q_{l t}(t / \sigma, 1) .
\end{aligned}
$$

Therefore, late-time production is unchanged if time is divided by $\sigma$ and production is multiplied by $\sigma$. This is the same scaling property displayed in Eq. (38) if we take $\sigma=s^{-2}$.

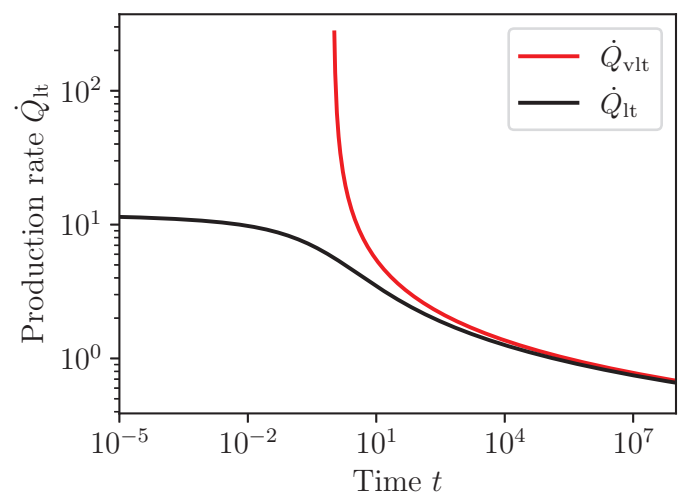

FIG. 16. Late-time and very-late-time production functions $\dot{Q}_{\mathrm{lt}}(t)$ and $\dot{Q}_{\mathrm{vlt}}$ for $\sigma=\sigma_{0}$. The late-time expression produces a value of $4 \pi$ for $t \ll 1$, but this is not meaningful; only $t \gg 1$ provides information about production rates.

In Fig. 16 we show $\dot{Q}_{\mathrm{lt}}(t)$. It drops less than two orders of magnitude over thirteen orders of magnitude in time. This means that the late-time production is almost linear, but with a coefficient of the linear relation that decreases slowly over exponentially long timescales. We also show the very latetime production function $\dot{Q}_{\mathrm{vlt}}(t)$, illustrating that behavior is essentially one over the log of time.

Because Eq. (40) describes the late-time behavior of all compact fracture networks, all of them can be described by an overall scale $\left(\rho_{0}\right)$ and the parameter $\sigma$. While $\sigma$ is defined to be dimensionless, it is connected to two important dimensional parameters. Note that $\sigma_{0}$ was defined in Eq. (32) so that with lattice spacing $a$ and for a square of size $\mathcal{V}_{\text {ext }}=65^{2} a^{2}$, $a^{2} \sigma=\mathcal{V}_{\text {ext }}$. Therefore $\sigma$ can be considered either to define the external volume $\mathcal{V}_{\mathrm{ext}}=a^{2} \sigma$ or a timescale we call the external time $\tau_{\mathrm{ext}}=\mathcal{V}_{\text {ext }} / \alpha$. The late-time behavior of flow to the fracture networks is like a black hole. All geometrical details drop away, and only the effective volume $\mathcal{V}_{\text {ext }}$ remains.

\section{Grid model}

We now set out to find simple functional forms to characterize collection networks with complicated geometries. The basic strategy is to solve some simple geometries exactly, and then use the scaling law in Eq. (38) to apply them to a broad range of other cases.

The idea we have in mind is illustrated in Fig. 17. We represent the stimulated volume $\mathcal{V}$ as a rectangular region subdivided into $N^{2}$ squares of side length $d$. It is clear that $\mathcal{V}$ and $d^{2}$ can be chosen completely independently. When their ratio is large, the network is highly ramified.

We assemble the functions needed to carry this out. For the interior, we need the interior production from a square.

The rate equation for flow to parallel plates was recorded in Eq. (28). Now we need the cumulative production, which is

$$
Q_{\text {plates }}(t)=\sum_{n=0}^{\infty} \frac{2}{(2 n+1)^{2}} \frac{4}{\pi^{2}}\left(1-e^{-(2 n+1)^{2} \pi^{2} t / 4}\right) .
$$




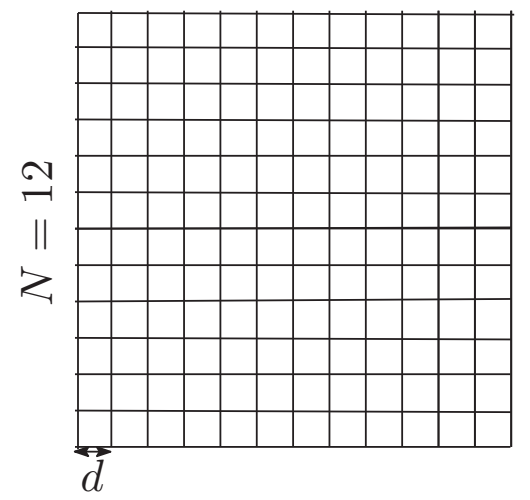

FIG. 17. Grid geometry with $N=12$.

According to the discussion in Sec. IIID2, the interior production of a unit square is given by

$$
\mathcal{Q}_{\text {int }}(t)=Q_{\text {plates }}(8 t) \text {. }
$$

The reason for the factor of 8 is that the solution was produced for plates at distance 2 from each other while here they are at distance 1 , and the fact that there are top and bottom boundaries doubles the rate at which gas is depleted. As shown in Fig. 9(b), deviations of the two-dimensional solution from the one-dimensional solution once it is sped up by 2 are negligible; indeed, the analytical solution for plates in Eq. (43) is also the analytical solution for interior production of a square once it has been scaled as described here.

In addition to a scaling function describing interior production, we need a scaling function describing exterior production. For this we use the exterior production function of the unit square, which is

$$
\mathcal{Q}_{\text {ext }}(t)=\frac{1}{65^{2}} Q_{\text {exterior }}^{\text {square }}\left(65^{2} t\right) .
$$

Here by $Q_{\substack{\text { square } \\ \text { exterior }}}$ we mean the exterior solution for the $65 \times 65$ square in Fig. 10. The factor $65^{2}$ rescales the solution to describe a unit square. While using a square to represent flow into the exterior of all structures is evidently a simple choice, without even taking into account aspect ratio, we have been guided in this direction by the fact that all geometric information but a single scale becomes irrelevant in the longtime limit.

We set down the quantities we will need to connect to dimensional version of the expressions:

(i) The diffusivity of the unperturbed background material is $\alpha$.

(ii) The exterior volume, exterior time, and exterior mass in place are

$$
\mathcal{V}_{\text {ext }}=\sigma a^{2}, \quad \tau_{\text {ext }}=\mathcal{V}_{\text {ext }} / \alpha, \quad \mathcal{M}_{\text {ext }}=\rho_{0} \mathcal{V}_{\text {ext }}
$$

(iii) The stimulated gas in place is

$$
\mathcal{M}=\mathcal{V} \rho_{0}=\rho_{0} d^{2} N_{x} N_{y}
$$

(iv) The interference time is

$$
\tau=d^{2} / \alpha
$$

The total gas production, from $N^{2}$ copies of production from interior squares, plus exterior, for the grid model is therefore $(t \equiv \tilde{t} / \tau)$

$$
\mathcal{Q}_{\text {grid }}(\tilde{t})=\mathcal{M} \mathcal{Q}_{\text {int }}(\tilde{t} / \tau)+\mathcal{M}_{\text {ext }} \mathcal{Q}_{\text {ext }}\left(\tilde{t} / \tau_{\text {ext }}\right) .
$$

In the particular case of a $65 \times 65$ square, our definitions of $\sigma_{0}$ and $\sigma$ in Eq. (33) ensure that $\mathcal{M}$ and $\mathcal{M}_{\text {ext }}$ are exactly equal. This exact equality will not hold for general structures, but the mass in place $\mathcal{M}$ and exterior mass in place $\mathcal{M}_{\text {ext }}$ should always be of the same order of magnitude. When equality holds, Eq. (49) is essentially the same as Eq. (14) in [14].

In short, the grid geometry, which we propose as a canonical form to capture complex fracture collection structures, has four parameters: stimulated original gas in place $\mathcal{M}$ and interference time $\tau$, which scale the interior solution along the horizontal and vertical axes, and the exterior mass in place exterior mass in place $\mathcal{M}_{\text {ext }}$ and exterior time $\tau_{\text {ext }}$. (gas density $\rho_{0}$ is assumed known). The exterior mass in place cannot vary far from the stimulated original gas in place, and thus most of the uncertainty about late times lies in the determination of $\tau_{\text {ext }}$. Finding this is equivalent to determining the unstimulated diffusivity $\alpha$.

In the next section we will explore a generalization of $\mathcal{Q}_{\text {int }}$ that accounts for a power-law distribution of interior squares, and we will then address the question of whether this constitutes an improvement.

\section{E. Why early-time production decay as $t^{-1 / 2}$ persists for complex geometries}

We address the question of whether fractal fracture networks might have power-law behavior at early times that differs from $t^{-1 / 2}$. Consider the following simple approximation to a decline curve; here $\theta(t)$ is a Heaviside function in time:

$$
\dot{Q}_{\text {early }}(t, \tau)=\frac{\theta(t) \theta(\tau-t)}{\sqrt{t \tau}} .
$$

This has the same behavior for small $t$ as the internal collection rate $\frac{1}{\tau} \dot{Q}_{\text {plates }}(t / \tau)$ in Eq. (28) and can therefore be used to examine how a probability distribution of interference times modifies the power laws of decline curves. Suppose that there is some probability distribution $P(\tau)$ that leads the early-time power law to change; that is,

$$
\begin{aligned}
\frac{1}{t^{\beta}} & =\int_{-\infty}^{\infty} d \tau P(\tau) \frac{\theta(t) \theta(\tau-t)}{\sqrt{t \tau}} \\
& \Rightarrow \frac{1}{t^{\beta-1 / 2}}=\theta(t) \int_{t}^{\infty} d \tau P(\tau) / \sqrt{\tau} .
\end{aligned}
$$

Restricting attention to positive $t$, differentiate with respect to time. Then

$$
(\beta-1 / 2) \frac{1}{t^{\beta+1 / 2}}=P(t) / \sqrt{t} .
$$

Note that since a probability distribution must be positive, there only exists a way to obtain the power law $t^{-\beta}$ if $\beta>1 / 2$. In this case, one has a normalized distribution

$$
P(\tau)=(1-\beta) \tau_{m}^{\beta-1} \tau^{-\beta} \theta\left(\tau_{m}-\tau\right),
$$


where $\tau_{m} \equiv 2 \tau$ is the maximum value allowed for the distribution. This corresponds to a collection network of finite size, which must present an upper bound on $\tau$.

We have that

$$
\begin{aligned}
\int_{0}^{\tau_{m}} d \tau P(\tau) \dot{Q}_{\text {early }}(t, \tau) & =\frac{1}{\sqrt{t}} \int_{t}^{\tau_{m}} d \tau(1-\beta) \frac{\tau_{m}^{\beta-1}}{\tau^{\beta+1 / 2}} \\
& =\frac{(1-\beta) \tau_{m}^{\beta-1}}{(\beta-1 / 2)}\left(t^{-\beta}-\tau_{m}^{-(\beta-1 / 2)} / \sqrt{t}\right) .
\end{aligned}
$$

Thus if $1>\beta>1 / 2$, the short-time behavior goes as $t^{-\beta}$, while if $\beta<1 / 2$ the short-time behavior continues to go as $1 / \sqrt{t}$. In other words, when a fracture network is fractal with a distribution of interference times $\tau$ going as $\tau^{-\beta}$ then the fractal geometry changes the observed power law of gas production when $\beta>1 / 2$, but for $\beta<1 / 2$ the early-time power law for production of gas is unaffected by the fractal geometry.

What happens if we now return to Eq. (28) and integrate over this distribution with the power-law distribution of interference times $\tau$ we just obtained? We obtain a new family of decline curves depending upon the additional parameter $\beta$ :

$$
\begin{aligned}
\dot{\mathcal{Q}}_{\text {int }}\left(t, \tau_{m}, \beta\right) & \equiv \int_{0}^{\tau_{m}} d \tau P(\tau, \beta) \frac{\dot{Q}_{\text {plates }}(t / \tau)}{\tau} \\
& =2(1-\beta) \sum_{n=0}^{\infty} \tau_{m}^{\beta-1}\left(\delta_{n} t\right)^{-\beta} \Gamma\left(\beta, \frac{\delta_{n} t}{\tau_{m}}\right),
\end{aligned}
$$

where

$$
\delta_{n}=(2 n+1)^{2} \pi^{2} / 4 .
$$

Here $\Gamma(\beta, x)$ is the upper incomplete Gamma function. Integrating over time yields a cumulative production curve

$$
\begin{aligned}
& \dot{\mathcal{Q}}_{\text {int }}(t / \tau, \beta) \\
& \quad=\sum_{n=0}^{\infty} \frac{2}{\delta_{n}}\left(1-e^{\delta_{n} t / 2 \tau}+\left(\delta_{n} t / 2 \tau\right)^{1-\beta} \Gamma\left(\beta, \frac{\delta_{n} t}{2 \tau}\right)\right) .
\end{aligned}
$$

In this last expression we replaced $\tau_{m}$ with $2 \tau$. This has the effect of making the extended scaling function with $\beta$ close to the old one without $\beta$ for those $\beta<1 / 2$ for which the earlytime behavior is similar. The production rate and cumulative production of the curves defined in Eq. (57) are displayed in Fig. 18. For $1 / 2<\beta<1$, the decline curve in Eq. (56) decays for early times as $t^{-\beta}$ and the cumulative production curve grows for early times as $t^{1-\beta}$. However, for $\beta<1 / 2$, the decline curve goes as $t^{-1 / 2}$ and the cumulative production curve increases as $t^{1 / 2}$. It turns out that this latter case is what is needed to account for the behavior of the model systems. In fact, the production of our multiscale systems is best fit for $-1<\beta<0$. This, we suggest, is the fundamental reason that the measured decline curves from stimulated wells display the $t^{-1 / 2}$ power law so universally, despite the fact that the true collection geometry is much more complicated than the models where the computations are usually done.

Note that since our calculations in this section rely only on a distribution of interference times $\tau$, they are not limited to two dimensions. They will apply equally well to cases
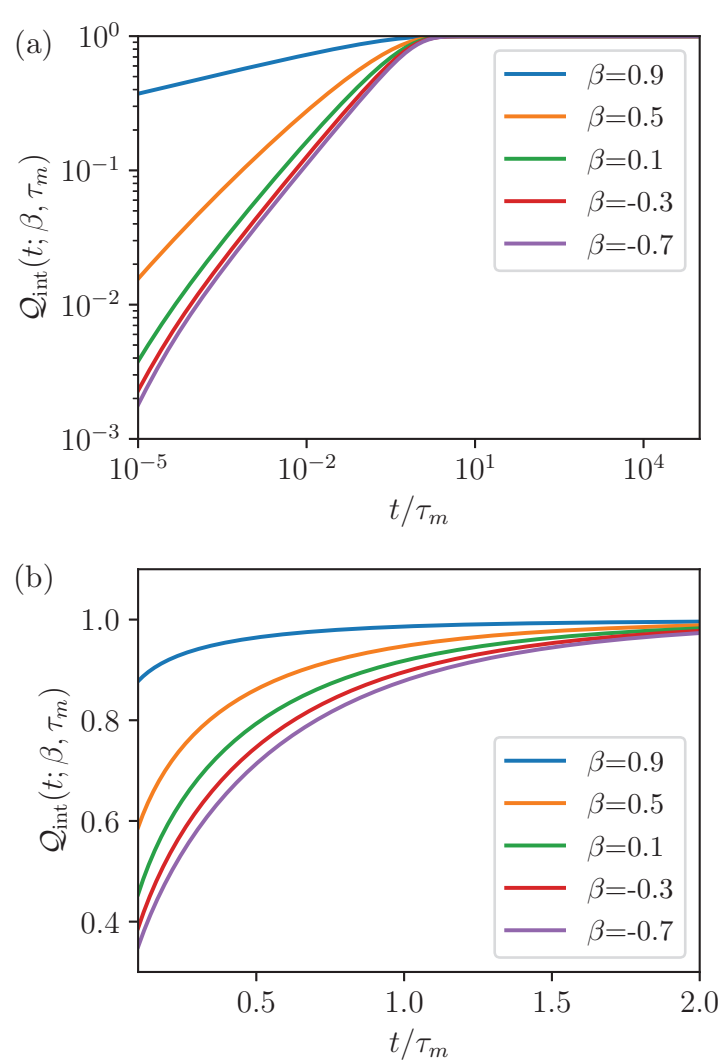

FIG. 18. Plots of cumulative production when the probability of interference time $\tau$ goes as $\tau^{\beta}$ up to a maximum interference time $\tau_{m}$. Curves are ordered with $\beta=0.9$ on top and $\beta=-0.7$ on bottom. (a) As $\beta$ drops below 0.5 the early-time asymptotic behavior of $\mathcal{Q}_{\text {int }}(t, \beta)$ stops depending upon $\beta$. (b) However, the detailed shape of the cumulative production curve in the vicinity of $t \approx \tau_{m}$ does depend upon $\beta$, and this does affect attempts to provide good phenomenological fits to exact solutions of model systems.

where three-dimensional fracture networks divide space up into orthorhombic regions

It would be interesting to inquire into the relationship between a distribution of fracture lengths and the distribution of interference times. We have carried out a preliminary analysis of this topic, but do not report on it here.

\section{F. Multiscale model}

Figure 19 shows a rectangular region with a power-law distribution of fractures. We use this as a test of the methods

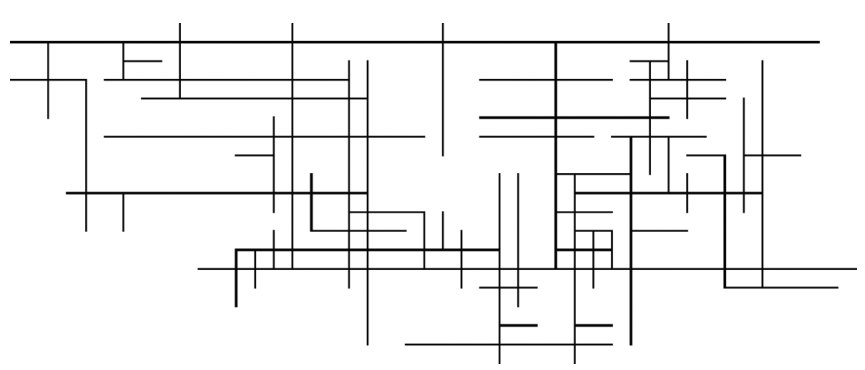

FIG. 19. Multiscale rectangular network used as test case. 
developed to this point, in an instance where we can compute the solution for production exactly, and we also know the geometry exactly, but the geometry differs from the grid model in Fig. 17. The lengths of the fractures are chosen from a power-law distribution with $e=1.2$ as in Eq. (1). The structure deliberately is constructed without a fine scale and thus is not very refined. The smallest distance between fractures is 10 lattice spacings (achieved because after construction, the network was scaled up by a factor of 10). This is done to limit the lattice effects so that they are evident only for times shorter than a tenth of the dominant interference time. While the resulting network is irregular, it is not ramified enough for one to say it is a good realization of a power-law distribution. It was as large as was feasible, however, for the exploratory analyses of this study. Several other statistically equivalent structures were studied as well to ensure that the results are robust, but this is the only one we will discuss in detail.

The convex hull of the fracture network has a (twodimensional) volume of $6.54 \times 10^{4}$. From the exact solution for gas flow we extract the external time $\tau_{\text {ext }}=a^{2} \sigma / \alpha$ by fitting to the asymptotic form on the right-hand side of Eq. (31). The relation $a^{2} \sigma=\mathcal{V}_{\text {ext }}$ gives the external volume of the structure, and we obtain $\mathcal{V}_{\text {ext }}=6.39 \times 10^{4}$. It is reassuring that the completely geometrical value from the convex hull is the same within around $10 \%$ as a value obtained from fitting long-time behavior.

We compute gas production of this fracture network, and then examine it in two phases. In the first phase we examine the complete production history, up to arbitrarily large times, and fit this using ideas from the previous sections. In addition to the form in Eq. (49) we also examine the following scaling form:

$$
\mathcal{Q}_{\text {grid } \beta}(\tilde{t})=\mathcal{M} \mathcal{Q}_{\text {int }}(\tilde{t} / \tau, \beta)+\mathcal{M}_{\text {ext }} \mathcal{Q}_{\text {ext }}\left(\tilde{t} / \tau_{\text {ext }}\right) .
$$

This is the same as Eq. (49) except that the internal solution for the stimulated volume now involves the generalized function from Eq. (57) depending upon $\beta$. We introduce $\beta$ reluctantly because each new parameter carries with it the possibility of better fits that come at the cost of reduced robustness in the procedure. We find that the quality of the fit without taking $\beta$ into account is worse, and a formal criterion (Akaike Information Criterion) says that the models with $\beta$ are better. We illustrate the accuracy of the model fit in Figs. 20 and 21. The first of these figures shows the production rate $\dot{Q}$ and cumulative production $Q$ comparing the exact result with the best model fit. As we did in Fig. 13(b), we use the subscript "total" to indicate the sum of interior and exterior solutions to obtain the complete exact result. The only visible deviations occur at early times where lattice effects are present. In order to obtain a more precise sense of the quality of the fit, in Fig. 21 we compute the slope of the log-log cumulative production depicted in Fig. 20(b). This means plotting $d \ln Q / d \ln t$, and it appears in Fig. 21. Figure 21(b) compares the exact result with the parametrization that excludes the fractal dimension $\beta$, while Fig. 21(a) shows the improvement in the fit when $\beta$ is included as a fitting parameter.

Nevertheless, our final judgment is that including the fractal dimension $\beta$ is not helpful enough to justify inclusion. This is based on two observations. The first is that we asked if the geometrical distribution of collection regions for networks
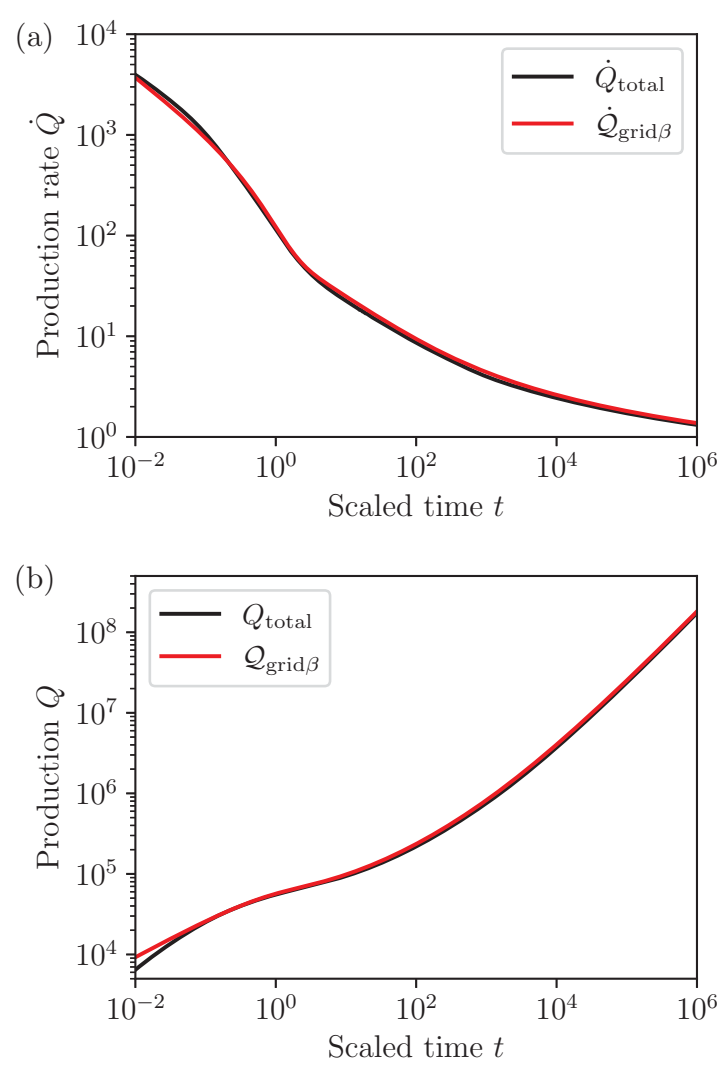

FIG. 20. Fits of the model in Eq. (58) to the exact production rate and cumulative production for the structure in Fig. 19. (a) Production rate, comparing the exact result $\dot{Q}_{\text {Total }}$ to the parameterized $\dot{\mathcal{Q}}_{\text {grid } \beta}$. (b) Cumulative production, comparing the exact result $Q_{\text {Total }}$ to the parameterized $\mathcal{Q}_{\text {grid } \beta}$.

such as the one in Fig. 19 corresponds to the value of $\beta$ that comes from a fit. The answer is that while the two are not incompatible, we are not in a position to say that the power-law distribution of internal collection regions correlates well with the exponent $\beta$ obtained from the fitting procedure. For example, based on a geometric count of the numbers of gas molecules diffusing distance $d$ (note $\tau=d^{2} / \alpha$ ) to reach a fracture, we estimate $\beta \approx 0 \pm 0.25$, while the fitting process gives best-fit $\beta=0.4$ for the fits in Figs. 20 and 21 .

A second approach to deciding whether or not to include $\beta$ comes when one tries to mimic the process of fitting to data that occurs in real life as time unfolds. We graph this process in Fig. 22. To prepare this figure, we truncate the exact solution $Q_{\text {Total }}$ at a value $t_{\mathrm{m}}$ and fit over this limited time interval to the model in Eq. (58). We let $t_{\mathrm{m}}$ advance and carry out the fits as it gets larger and larger. We carry out this process both for the parameterized form $\mathcal{Q}_{\text {grid }}$ that does not include the power-law exponent $\beta$ and the form in Eq. (58) that does. In the test cases we have examined, there are instances where including $\beta$ gives more accurate estimates of the parameters at earlier times. Yet this is not always the case. As shown in Fig. 22(b), when the system has reached the age of the interference time $\tau$ (a fact that would not in reality be known at the time) the estimate of the diffusivity $\alpha$ without using $\beta$ is too high by a factor of 10 . By contrast, at this point the model employing $\beta$ is too high by a factor 

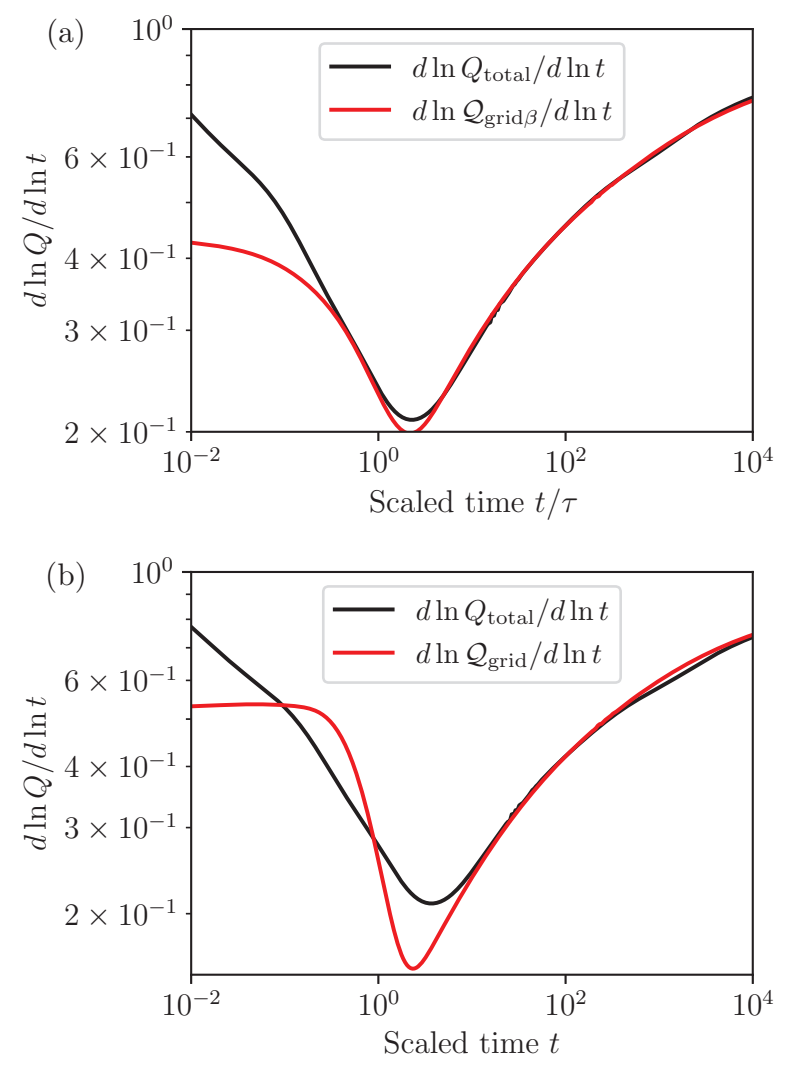

FIG. 21. Slope of log-log production plot, comparing models with parameterized fits. (a) Slope of log-log cumulative production including fractal exponent $\beta$ from Eq. (58). (b) Slope of log-log cumulative production without fractal exponent $\beta$ in Eq. (49).

of 2. When the time reaches $5 \tau$ (about as far in time as any profitable wells in the real world have proceeded) the model without $\beta$ provides an estimate of diffusivity $\alpha$ that is high by a factor of 2 , while the model with $\beta$ is within $90 \%$ of the correct answer. But this is fortuitous, since the estimate of $\alpha$ then drops to $70 \%$ of the true value before eventually converging to an accurate final estimate when $t_{\mathrm{m}} \approx 100 \tau$ (taking a typical value of $\tau=3$ years, we would have to wait for the year 2300 to check this prediction thoroughly with field data). Furthermore, the estimate of $\tau$ is better in the model without $\beta$ and the estimate of $\mathcal{M}$ is comparable in the two cases.

These estimates will be degraded further if stochastic values are added to the production measurements, and these are present in all real data sets. On the other hand, the estimates will improve if $\alpha$ is treated as a field-wide physical constant, rather than a parameter to be determined well by well. Thus further effort is appropriate if these methods are to turn into a practical tool.

\section{DISCUSSION}

When we began this work, our motivation was to learn the extent to which the time history of gas coming from a well could be used to infer the geometry of the transport network feeding the well.

To address this question, we constructed and solved model systems. Our geometry consists of an infinite square lattice
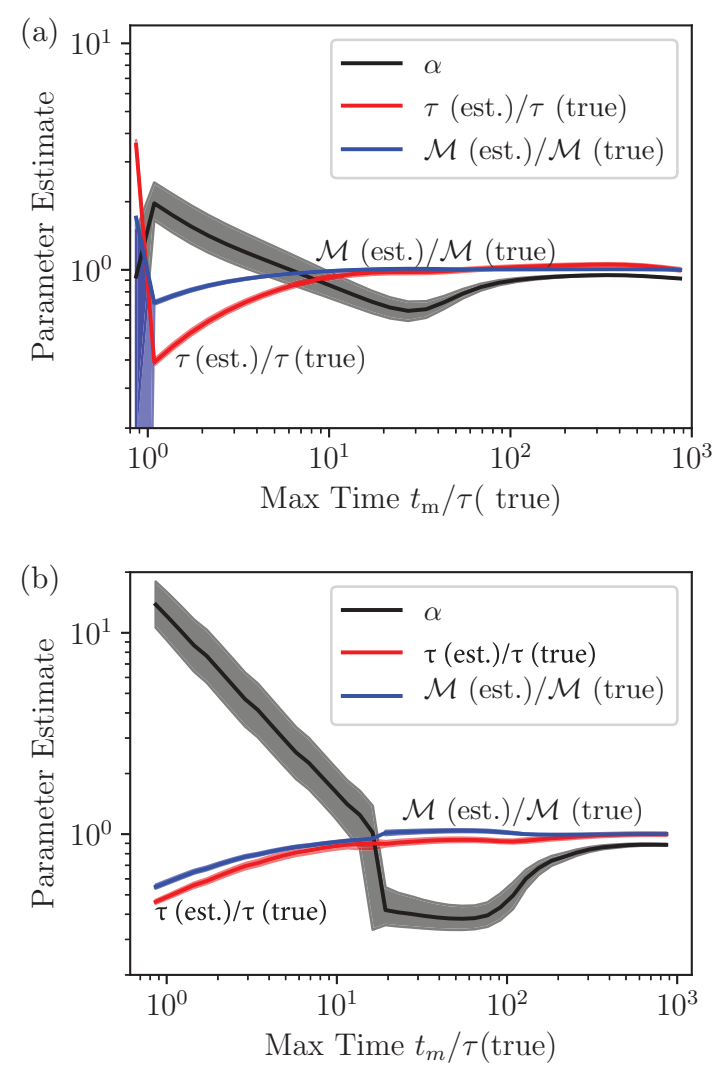

FIG. 22. Comparison of fits obtained to production data as maximum available time increases. The bands around each line show one- $\sigma$ uncertainties obtained from the statistical routine conf_interval in the package lmfit [22]. The uncertainties are almost always underestimates. (a) Estimates of model parameters, including long-time behavior, making use of fractal exponent $\beta$. (b) Estimates of model parameters, including long-time behavior, without making use of fractal exponent $\beta$.

with uniform initial gas saturation, populated with a network of absorbing sites. The formalism we described in this work makes it possible to obtain an analytical solution for the time behavior of the rate of mass transport into the absorbers and out of the domain. The solution can be obtained for any finite network of absorbers on a square lattice and is valid over very long times.

Our main contributions are the following:

(1) We implemented two separate methods to solve the model problem. The first of these is a kinetic Monte Carlo method that has the advantage of treating short distances as a continuum, but has the disadvantage of providing a solution with a stochastic component and subject to finite-size effects at large scales. The second of these is a Green's function method that has the advantage of providing an exact solution without finite-size effects at large scales, but the disadvantage of lattice effects at short scales.

(2) To implement the Green's function method we had to find a numerical procedure capable of computing unperturbed lattice Green's functions for ranges of arguments that appear not to have been achieved previously.

(3) By comparing our two methods, we determined that an apparent power law visible in the solutions obtained from 
kinetic Monte Carlo is a finite-size effect. We also determined that early-time results from the Green's function method showed unrealistic lattice effects unless we expanded structures so that the smallest structures of interest were separated by many lattice spacings.

(4) Late-time gas production takes a universal form scaled by two parameters that corresponds to radial diffusion of gas from far away towards an absorbing square. One of the parameters is a timescale, and the other parameter is the diffusivity of the unperturbed medium.

(5) Fractal geometry of the collection network affects early-time production. The nature of the effect depends on the power law governing interference times. In one regime, the gas production rate at early times goes as $t^{-\beta}$, where $\beta>1 / 2$. In the other regime, which is the one that describes realistic structures, the production rate at early times goes as $t^{-1 / 2}$, independent of the power law describing the fractal fracture network.

(6) In establishing a practical method to fit both early- and late-time production for our model systems from production data obtained as early as possible, we found it best to use four parameters: a timescale and a volume characterizing latetime production (together these yield the diffusivity of the unfractured rock), and a timescale and volume characterizing early-time production. If the diffusivity of unfractured rock can be treated as a known physical quantity, the fitting task drops down to finding three parameters.

In [14] we described the practical implications of determining the long-time production of hydrofractured wells. The assumptions of that paper we now see have been borne out, although they were based on intuition rather than on the derivations we have just provided. It is worth summarizing some of the previous results because they explain the practical significance of the current results. The amount of gas coming from a single well in 30 years can be $30 \%-50 \%$ greater than is predicted by fits to the early-time solution. The distance around the well from which this gas comes impacts the economics of filling in the field with additional wells. This distance (Fig. 15 in [14]) is estimated to be 50 to $100 \mathrm{~m}$. That is, the return over 30 years comes from the depletion of a region that is quite close to the well.

Hydrofracturing is such a recent process that no data are available to check whether theoretical expectations about latetime behavior are correct, and whether fitting procedures are accurate. The model systems in this paper provide a theoretical laboratory where all these points can be checked. Actual field conditions introduce many elements that go beyond the models; three-dimensional structures, production fluctuations, and multiphase flow are some of the most important. But learning to make predictions correctly for the ideal two-dimensional models explored here is a critical first step towards a physically accurate description of the decline of unconventional gas and oil wells.

\section{ACKNOWLEDGMENTS}

Partial support for this work was provided by the US National Science Foundation through Award No. 1810196, Fracture and Transport Problems for Inhomogeneous Brittle Materials, and by a Competitive Research Grant from
KAUST, "Numerical and Experimental Investigation of Gas Distribution, Complex Hydrofractures and the Associated Flow in the Jafurah Basin Shales: Fundamentals to Applications.” The opinions expressed in this work are not necessarily shared by the National Science Foundation.

\section{APPENDIX A: EQUATION OF MOTION FOR EXTERIOR PROBLEM}

Our goal is to find an equation of motion for $|\rho(t)\rangle$ that is identical to Eq. (6) in the exterior region $O$ (Fig. 7), but that leaves the gas density for all interior sites always unchanged. This solution can then be added to the solution for the interior to obtain the total gas flow.

In order to accomplish this task, let us return to the equation of motion in Eq. (6) and modify it. When there are no absorbers we have

$$
\dot{\rho}_{j}(t)=\sum_{j^{\prime}} k_{j j^{\prime}}\left[\rho_{j^{\prime}}(t)-\rho_{j}(t)\right]=\sum_{j^{\prime}} H_{j j^{\prime}}^{0} \rho_{j^{\prime}},
$$

with

$$
H_{j j^{\prime}}^{0}=k_{j j^{\prime}}-4 \delta j j^{\prime} .
$$

Here $j$ ranges over the entire infinite square lattice. Next suppose there is a set of absorbers indexed by $m$ that forms the outer boundary of a fracture network (space $M$ in Fig. 7) and that the neighbors of these sinks on the outside of the network are labeled with $m^{\prime \prime}$ (space $M^{\prime}$ in Fig. 7). Then the drainage from a site $m^{\prime \prime}$ to its neighboring absorber $m$ can be described by adding a term to the right hand side of Eq. (A1):

$$
\begin{aligned}
\dot{\rho}_{m^{\prime}}(t)= & \sum_{j^{\prime}} k_{m^{\prime} j^{\prime}}\left[\rho_{j^{\prime}}(t)-\rho_{m^{\prime}}(t)\right] \\
& -\sum_{m \in M} k_{m^{\prime} m} \rho_{m}(t) .
\end{aligned}
$$

The last term produces the same effect as if the gas density at all the absorbers were zero, because it cancels any nonzero $\rho_{m}$ out of the equation for $\rho_{m^{\prime}}$. If this were the only term added to the equation of motion, then gas density at the absorber $m$ would change, and this would in turn cause gas flow to commence from the interior of the structure. This can be prevented by adding an additional term to the equation of motion for density at the absorber $m$ which precisely cancels any flow along the bond connecting $m$ and its exterior neighbors $m^{\prime}$ :

$$
\begin{aligned}
\dot{\rho}_{m}(t)= & \sum_{j^{\prime}} k_{m j^{\prime}}\left[\rho_{j^{\prime}}(t)-\rho_{m}(t)\right] \\
& -\sum_{m^{\prime} \in M^{\prime}} k_{m m^{\prime}}\left[\rho_{m^{\prime}}(t)-\rho_{m}(t)\right] .
\end{aligned}
$$

Putting together these terms produce the desired effect. All the sites in the interior are ringed around by absorbers on the boundary. Their densities cannot change because the gas density on the absorbers does not change, and the density on the absorbers cannot change because the term that couples them to the exterior is zeroed out in Eq. (A4). However, the external neighbors of the absorbers send gas to them as if the absorbers are empty, according to Eq. (A3), and as all the external sites are coupled together, gas flows into the boundary 
according to the solution to the exterior problem. Returning to operator notation, the Hamiltonian governing the exterior problem is given by

$$
\hat{H}-\hat{H}^{0} \equiv \hat{H}^{1}=\sum_{\substack{m \in M \\ m^{\prime} \in M^{\prime}}} k_{m m^{\prime}}\left(|m\rangle\left\langle m|-| m^{\prime}\right\rangle\langle m|-| m\rangle\left\langle m^{\prime}\right|\right) .
$$

Note that $\hat{H}^{1}$ is symmetric and is nonzero only in the space $N$ (Fig. 7) spanned by absorbers and their nearest neighbors connected to the exterior. Equation (A5) can be interpreted as a discrete operator for flux into the boundary of the collection network. The sum contains a term for every bond connecting an absorber to a site on the exterior.

\section{APPENDIX B: GREEN'S FUNCTION SOLUTION}

\section{Green's function definition}

We intend to find an exact solution of Eq. (6) for the Hamiltonian in Eq. (A5). Note that the full Hamiltonian is the sum of two pieces. The first of them, $\hat{H}^{0}$ is defined over an infinite-dimensional space but is analytically tractable. The second of them, $\hat{H}^{1}$ is not analytically tractable in the same way but is defined on a finite-dimensional space. The method of Green's functions lets us turn the whole problem into a matrix problem on a finite-dimensional space.

Adopt the convention that

$$
|\rho(\omega)\rangle=\int_{0}^{\infty} d t e^{-i \omega t}|\rho(t)\rangle=\sum_{\alpha} \frac{1}{i \omega-E_{\alpha}}|\alpha\rangle\left\langle\alpha \mid \rho_{\mathrm{i}}\right\rangle .
$$

For $t>0 \omega$ must have some negative imaginary component for the integral to converge. Then we have

$$
|\rho(\omega)\rangle=\hat{G}(i \omega)\left|\rho_{\mathrm{i}}\right\rangle
$$

with

$$
\hat{G}(E)=\frac{1}{E-\hat{H}}
$$

and

$$
|\rho(t)\rangle=\int_{-\infty}^{\infty} \frac{d \omega}{2 \pi} e^{i \omega t} \hat{G}(i \omega)\left|\rho_{\mathrm{i}}\right\rangle=\int_{-i \infty}^{i \infty} \frac{d E}{2 \pi i} e^{E t} \hat{G}(E)\left|\rho_{\mathrm{i}}\right\rangle .
$$

$\hat{G}(E)$ as defined in Eq. (B3) is Green's function, and the solution for $\rho(t)$ is obtained by integrating on a contour that traces out the imaginary axis.

\section{Unperturbed Green's function}

Consider the Hamiltonian $H^{0}$, Eq. (A2) for gas diffusion on an infinite two-dimensional lattice without any absorbers. Green's functions for this problem are extremely well known and described in many textbooks [21], although to the best of our knowledge they have not previously been computed in the general cases we will need. We briefly review the solution as we will need several of the expressions for the formal development that follows. The definition of the unperturbed Green's function is

$$
\hat{G}^{0}\left(E-\hat{H}^{0}\right)=1 .
$$

What are the eigenfunctions and eigenvalues of $\hat{H}^{0}$ ? They are just Fourier modes: Choose $\vec{k}$ in the first Brillouin zone for a Bravais lattice $\vec{R}$. For the unit square lattice used here, $\vec{k}=$ $2 \pi\left(n_{1}, n_{2}\right) / N$, where $N$ is the number of lattice sites and $n_{1}$ and $n_{2}$ are integers ranging from 0 to $N-1$,

$$
|\vec{k}\rangle=\frac{1}{\sqrt{N}} \sum_{\vec{R}} e^{i \vec{R} \cdot \vec{k}}|\vec{R}\rangle .
$$

Let $\vec{\delta}$ be the nearest neighbor vectors of each lattice site. Then the eigenvalue $E_{\vec{k}}$ is

$$
E_{\vec{k}}=-\sum_{\vec{\delta}}(1-\cos \vec{k} \cdot \vec{\delta})
$$

and

$$
\hat{H}^{0}|\vec{k}\rangle=E_{\vec{k}}|\vec{k}\rangle .
$$

Therefore for any initial condition $\rho(0)$

$$
\left|\rho_{0}(t)\right\rangle=\sum_{\vec{k}} e^{E_{\vec{k}} t}|\vec{k}\rangle\langle\vec{k} \mid \rho(0)\rangle .
$$

Green's function is given by

$$
\hat{G}^{0}=\sum_{\vec{k}} \frac{|\vec{k}\rangle\langle\vec{k}|}{E-E_{\vec{k}}} .
$$

When the lattice is uniformly filled with gas, it is in a state that is proportional to $|\vec{k}=0\rangle$. Because the energy $E_{\vec{k}}$ vanishes for $|\vec{k}=0\rangle$,

$$
\hat{G}^{0}(E)\left|\rho_{\mathrm{i}}\right\rangle=\frac{\left|\rho_{\mathrm{i}}\right\rangle}{E} \quad \text { and }\left\langle\rho_{\mathrm{i}}\right| \hat{G}^{0}(E)=\frac{\left\langle\rho_{\mathrm{i}}\right|}{E} .
$$

Expressions for matrix elements $\left\langle\vec{R}\left|\hat{G}^{0}\right| \vec{R}^{\prime}\right\rangle$ in one, two, and three dimensions for various Bravais lattices are available in the literature [21]. An explicit although computationally impractical expression for the square lattice with unit lattice spacing is

$$
\begin{aligned}
& \left\langle(0,0)\left|\hat{G}^{0}(E)\right|(x, y)\right\rangle \\
& =\frac{1}{(2 \pi)^{2}} \int_{-\pi}^{\pi} d k_{x} \int_{-\pi}^{\pi} d k_{y} \frac{e^{i k_{x} x+i k_{y} y}}{E+B-2 \cos k_{x}-2 \cos k_{y}},
\end{aligned}
$$

where $B=4$ is the number of nearest neighbors of each lattice site.

\section{Numerical method to compute unperturbed Green's functions}

Solution of the gas flow problem requires computation of the unperturbed lattice Green's function between arbitrary sites $x, y$. These are the matrix elements (here $\lambda<0$ )

$$
G_{x, y}(\lambda)=\lim _{\eta \rightarrow 0}\left\langle(0,0)\left|\hat{G}^{0}(\lambda-i \eta)\right|(x, y)\right\rangle .
$$

The fastest numerical procedure uses recursion relations [23]. First find $G_{00}$ and $G_{10}$ which are given by

$$
\begin{aligned}
z & =\lambda+4, \\
k & =z / 4, \\
k^{\prime} & =\sqrt{1-k^{2}},
\end{aligned}
$$




$$
\begin{aligned}
\operatorname{Im} G_{00} & =\frac{1}{2 \pi} K\left(k^{\prime}\right), \\
\operatorname{Re} G_{00} & =\operatorname{sgn}(z) \frac{1}{2 \pi} K(k) .
\end{aligned}
$$

Note that many numerical routines take the square of the argument given here for the complete elliptic integral of the first kind, $K$. For $G_{11}$, with $E$ the complete elliptic integral of the second kind,

$$
\begin{aligned}
K_{i} & =k K\left(k^{\prime}\right), \\
K_{r} & =k K(k), \\
E_{i} & =\frac{1}{k}\left[-E\left(k^{\prime}\right)+k^{2} E\left(k^{\prime}\right)\right], \\
E_{r} & =\frac{1}{k}\left[E(k)-k^{\prime 2} E(k)\right], \\
\operatorname{Im} G_{11} & =\frac{2}{\pi z}\left[\left(2 k^{2}-1\right) K_{i}-2 k^{2} E_{i}\right], \\
\operatorname{Re} G_{11} & =\frac{2}{\pi|z|}\left[\left(2 k^{2}-1\right) K_{r}-2 k^{2} E_{r}\right] .
\end{aligned}
$$

From these two matrix elements all the rest can be obtained by recursion. It is convenient to define

$$
g_{y}^{s}=G_{y+s, y} ; \quad G_{x, y}=g_{y}^{x-y} .
$$

Then all the diagonal elements of $G$ can be obtained from

$$
g_{y+1}^{0}=\frac{4 y}{2 y+1}\left(\frac{z^{2}}{8}-1\right) g_{y}^{0}-\frac{2 y-1}{2 y+1} g_{y-1}^{0} .
$$

Next use the recursion relation

$$
\begin{aligned}
\left(E-\hat{H}^{0}\right) \hat{G}^{0} & =1 \Rightarrow(\lambda+4) G_{x y}-G_{x+1, y}-G_{x-1, y}-G_{x, y+1} \\
-G_{x, y-1} & =\delta_{x, 0} \delta_{y, 0} .
\end{aligned}
$$

From this one can deduce the following:

$$
\begin{aligned}
& g_{0}^{1}=\frac{z g_{0}^{0}-1}{4}, \\
& g_{y}^{1}=\frac{z}{2} g_{y}^{0}-g_{y-1}^{1} \text { for } y \geqslant 1, \\
& g_{0}^{s}=z g_{0}^{s-1}-g_{0}^{s-2}-2 g_{1}^{s-2} \text { for } s \geqslant 2, \\
& g_{y}^{s}=z g_{y}^{s-1}-g_{y-1}^{s}-g_{y}^{s-2}-g_{y+1}^{s-2} \text { for } s \geqslant 2 \text { and } y \geqslant 1 .
\end{aligned}
$$

The papers introducing these recursion relations employ them for values on the order of $s \leqslant 5$. The reason larger values do not appear is that the recursions are exponentially unstable. The instability is particularly severe as $\lambda$ approaches the band edge $\lambda=0$. We found reports of effective methods for computation of the unperturbed lattice Green's function for large imaginary $\lambda$ [24], but those methods do not function for $\lambda$ approaching the band edge along the real axis. Integral formulations derived from Eq. (B12) are slow and inaccurate, particularly for lattice numbers $x$ and $y$ on the order of 100 , and $\lambda$ close to the band edge. Because the Green's functions behave as logarithms near the band edge, values as small as $\lambda \approx 10^{-2000}$ are needed in order to carry out the integral in Eq. (D5). The solution we adopted is to employ Eq. (B19) with high-precision arithmetic. For example, when $\lambda=10^{-2000}$, the computation is performed with 4000 places of precision. For $x$ and $y$ on the order of 100, the recursions require a minimum of 100 places of precision almost everywhere in the band. Once the Green's function matrix elements have been obtained, the rest of the computation can be carried out with ordinary double precision floating point numbers.

So far as we can tell, despite the fact that the Green's functions employed here have been studied for decades, this is the first occasion where they have actually been computed near the band edge for lattice hops larger than 10. We provide a few remarks on numerical implementation. We used mpmath [25], SciPy [26], and Python [27]. We were concerned that the computation of Green's functions was very slow, and therefore rewrote the arbitrary precision Green's functions routines in C using arb [28], which we then linked to Python and SciPy using SWIG [29]. While this did speed up the computation of Green's functions by a factor of 100 , that turned out not to be the rate-limiting step. The unexpectedly time-consuming step was loading the matrix $\left\langle v^{\prime}\left|\hat{G}^{0}\right| v\right\rangle$ after all necessary matrix elements had been computed. For example, consider a problem in a $100 \times 100$ spatial domain. All Green's functions $G_{x y}$ need to be computed where $0 \leqslant x \leqslant y \leqslant 100$. This means finding around 5000 values making use of Eq. (B19). By contrast the state vectors $v$ range over 10000 values, and therefore the matrix $\left\langle v^{\prime}\left|\hat{G}^{0}\right| v\right\rangle$ has $10^{8}$ entries. Each of these is drawn from the 5000 Green's functions that were previously computed, but simply looking them up and inserting them turned out to take much more time in Python than computing them to begin with. No rapid way to perform this task in SciPy was found so this portion of the code was also rewritten in $\mathrm{C}$. Once this was done, the rate-limiting step became finding $|\mathcal{T}\rangle$ from the linear system in Eqs. (C7). SciPy does this efficiently using routines from LINPACK [30].

\section{APPENDIX C: T MATRIX SOLUTION}

Let $\hat{G}$ be Green's function for the full Hamiltonian for the exterior problem $\hat{H}=\hat{H}^{0}+\hat{H}^{1}$ in Eq. (A5). We now can compute the Green's function $\hat{G}^{0}$ that corresponds to the unperturbed Hamiltonian $\hat{H}^{0}$ and starting with this we want to find an expression that relates it to the full problem.

The $T$ matrix corresponding to a Green's function is defined by the following formal relation between $\hat{G}$ and $\hat{G}^{0}$ :

$$
\hat{G}=\hat{G}^{0}+\hat{G}^{0} \hat{T} \hat{G}^{0}
$$

Standard manipulations found for example in [21] give an expression for the $T$ matrix as an infinite series:

$$
\hat{T}=\hat{H}^{1}+\hat{H}^{1} \hat{G}^{0} \hat{H}^{1}+\hat{H}^{1} \hat{G}^{0} \hat{H}^{1} \hat{G}^{0} \hat{H}^{1} \cdots
$$

This makes clear that $\hat{T}$ like $\hat{H}^{1}$ is nonzero only in the space $N$ of absorbers and their exterior neighbors (Fig. 7). The essential point is this: we have a diffusion problem defined on an infinite two-dimensional lattice. Its solution can be expressed in terms of the unperturbed Green's function $\hat{G}^{0}$ which is defined on the infinite lattice, and $\hat{T}$, which is defined in a finite-dimensional space specified by the finite collection of absorbers. This turns an infinite-dimensional problem into a finite-dimensional one. 
One can write for the $T$ matrix

$$
\hat{T}=\hat{H}^{1}+\hat{H}^{1} \hat{G}^{0} \hat{T} .
$$

Thus $\hat{T}$ is determined by solving the matrix equation

$$
\sum_{v^{\prime \prime}}\left\langle v\left|\left(1-\hat{H}^{1} \hat{G}^{0}\right)\right| v^{\prime \prime}\right\rangle\left\langle v^{\prime \prime}|\hat{T}| v^{\prime}\right\rangle=\left\langle\nu\left|\hat{H}^{1}\right| v^{\prime}\right\rangle .
$$

Here the indices $v, v^{\prime}$, and $v^{\prime \prime}$ belong to $N$. To compute gas production from Eq. (14) one does not need the complete $T$ matrix, but just sums over all its matrix elements. As we will see, it suffices to find

$$
\mathcal{T}_{\nu} \equiv \sum_{v^{\prime}}\left\langle\nu|\hat{T}| \nu^{\prime}\right\rangle=\langle\nu \mid \mathcal{T}\rangle .
$$

Also define

$$
\mathcal{H}_{v} \equiv \sum_{v^{\prime}}\left\langle\nu\left|\hat{H}^{1}\right| v^{\prime}\right\rangle \equiv\langle\nu \mid \mathcal{H}\rangle .
$$

Then the equation satisfied by the relevant part of the $T$ matrix is

$$
\sum_{v^{\prime}}\left\langle v\left|\left(1-\hat{H}^{1} \hat{G}^{0}\right)\right| v^{\prime}\right\rangle \mathcal{T}_{\nu^{\prime}}=\mathcal{H}_{\nu},
$$

which can be written as

$$
\left(1-\hat{H}^{1} \hat{G}^{0}\right)|\mathcal{T}\rangle=|\mathcal{H}\rangle
$$

\section{APPENDIX D: GAS PRODUCTION}

\section{Derivation from Green's functions}

The relation between the full Green's function and the amount of gas produced at time $t$ is

$$
Q(t)=\left\langle\rho_{\mathrm{i}} \mid \rho_{\mathrm{i}}\right\rangle-\int_{-\infty}^{\infty} \frac{d \omega}{2 \pi} e^{i \omega t}\left\langle\rho_{\mathrm{i}}|\hat{G}(i \omega)| \rho_{\mathrm{i}}\right\rangle .
$$

Use Eq. (C1) to eliminate $\hat{G}$. It gives

$$
\begin{aligned}
Q(t)= & \left\langle\rho_{\mathrm{i}} \mid \rho_{\mathrm{i}}\right\rangle-\int_{-\infty}^{\infty} \frac{d \omega}{2 \pi} e^{i \omega t}\left\langle\rho_{\mathrm{i}}\right|\left[\hat{G}^{0}(i \omega)\right. \\
& \left.+\hat{G}^{0}(i \omega) \hat{T}(i \omega) \hat{G}^{0}(i \omega)\right]\left|\rho_{\mathrm{i}}\right\rangle .
\end{aligned}
$$

Using Eq. (B11) (and recalling that the Fourier transform of $1 / i \omega$ is $2 \pi$ for $t>0$ ) the first two terms cancel, so that the gas produced is

$$
Q(t)=\int_{-\infty}^{\infty} \frac{d \omega}{2 \pi} e^{i \omega t} \frac{1}{\omega^{2}} \sum_{\nu \nu^{\prime}}\left\langle\nu|\hat{T}(i \omega)| v^{\prime}\right\rangle .
$$

We indicate the sum over $v$ explicitly to emphasize that we are now working in the finite-dimensional space $N$, rather than the infinite two-dimensional lattice on which $\left|\rho_{\mathrm{i}}\right\rangle$ is defined. Taking the time derivative, gas production $\dot{Q}$ is

$$
\dot{Q}(t)=-\int_{-i \infty}^{i \infty} \frac{d E}{2 \pi i} e^{E t}\left[\frac{1}{E} \sum_{\nu v^{\prime}}\left\langle\nu|\hat{T}(E)| v^{\prime}\right\rangle\right] .
$$

All the poles of $\hat{T}$ are on the negative real axis, starting just a little bit below 0 , and proceeding down to -8 . One can perform the integral by deforming the contour to run just below the real axis from $-\infty$ up to zero, crossing over the top and running down the real axis for positive imaginary part

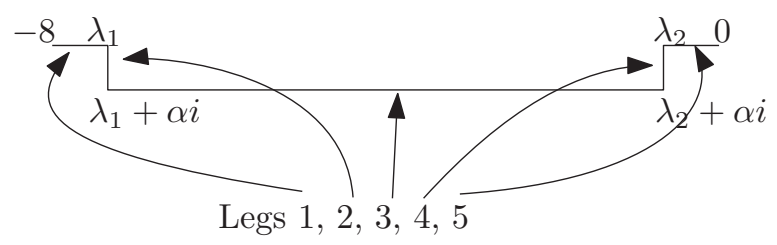

FIG. 23. Integration contour used for Eq. (D5). The values used in computation were $\lambda_{1}=-8+10^{-5}, \alpha=-0.1, \lambda_{2}=-10^{-5}$.

from 0 to $-\infty$. This leads to an expression for energies with vanishing negative imaginary part $\eta$ :

$$
\begin{aligned}
\dot{Q} & =-\lim _{\eta \rightarrow 0} \int_{-8}^{0} \frac{d \lambda}{\pi \lambda} e^{\lambda t} \operatorname{Im}\left[\sum_{\nu \nu^{\prime}}\left\langle\nu|\hat{T}(\lambda-i \eta)| \nu^{\prime}\right\rangle\right] \\
& =-\lim _{\eta \rightarrow 0} \int_{-8}^{0} \frac{d \lambda}{\pi \lambda} e^{\lambda t} \operatorname{Im} \sum_{\nu} \mathcal{T}_{\nu}(\lambda-i \eta) \\
& =-\int_{-8}^{0} \frac{d \lambda}{\pi \lambda} e^{\lambda t} \operatorname{Im}\left\langle\rho_{\mathrm{i}} \mid \mathcal{T}(\lambda)\right\rangle .
\end{aligned}
$$

When $\mathcal{T}_{v}(\lambda)$ has a real argument, it will be understood to be $\lim _{\eta \rightarrow 0} \mathcal{T}_{v}(\lambda-i \eta)$. Eq. (D5) provides an explicit expression relating the remaining gas to a computation performed only within the subspace of absorbers and their nearest neighbors.

The numerical strategy we followed at first was to compute the contributions from the discrete spectrum (the interior sites) from Eq. (15) and the contributions from the continuous spectrum (external sites) from Eq. (D5). The integrals were extremely slow to converge because as shown in Fig. 12(a) the integrand consists of thousands of narrow peaks. These are produced by cavities in the structure that are nearly, but not completely, closed regions with discrete levels. It proved greatly preferable to deform the integration contour as shown in Fig. 23. With this contour, integrals converge much more rapidly. The numerical values in the caption of Fig. 23 were obtained by optimizing convergence time for some simple test cases.

In our previous publication [13] we had to conduct a complicated process to deal with discrete modes we found during the solution of this exterior problem. These problems were eliminated once we defined the perturbing Hamiltonian through Eq. (A5) rather than a Hamiltonian used in the previous publication.

A final definition and change of variables puts the gas production rate into the most numerically tractable form for the portion of the contour where $\lambda$ lies on the real axis. Define

$$
\begin{gathered}
f \equiv-\ln (-\lambda) \\
\mathscr{T}(f) \equiv \lim _{\eta \rightarrow 0} \operatorname{Im} \sum_{\nu} \mathcal{T}_{v}\left(-e^{-f}-i \eta\right) .
\end{gathered}
$$

Then the fifth leg of the contour in Fig. 23, which requires caution to compute accurately and completely dominates the integral at large times, is

$$
\dot{Q}^{(5)}=\frac{1}{\pi} \int_{-\ln \lambda_{2}}^{\infty} d f \exp \left(-t e^{-f}\right) \mathscr{T}(f) .
$$




\section{Additional comments on numerical method}

For any given time the integral in Eq. (D5) is performed with the SciPy routine quad [31], which evaluates the integrand at a set of quadrature points. Almost all the numerical time is spent computing $|\mathcal{T}\rangle$. For the computation at $t=0$ on the order of 2000 function evaluations are necessary. It appears that to carry out the computation for successive values of time $t$ will require repeating this computation from scratch, but that is not the case. The quadrature routine selects mainly the same evaluation points as $t$ increases. Therefore the strategy is to store $\operatorname{Im}\left\langle\rho_{\mathrm{i}} \mid \mathcal{T}(\lambda)\right\rangle$ every time it is evaluated in a dictionary keyed to the complex argument $\lambda$. As the quadrature proceeds for increasing values of $t$, most of the time it terminates without needing any new function evaluations at all. If the quadrature decides it needs new points, it selects them, they are computed, and added to the dictionary. The result is that the most time-consuming step is to compute the production rate at $t=0$ (where the exact answer is known in advance), and the computation time for all remaining times put together is less. In general we computed the production rate for around 600 different times equally spaced on a $\log$ scale (apart from $t=0$ ) up to $t=10^{10}$. It turns out that the value of time $t$ for which the computations are most numerically challenging is around $t=1000$. At this point there are very rapid oscillations of the integrand on the fourth leg of the integration contour in Fig. 23. The integration routine has difficulty converging, and some very slight glitches are visible in some of the plots of production rate versus time. For example, there are slight wobbles in the black curves in Fig. 21. For much larger values of $t$ only the fifth leg of the contour is important, and as on this portion of the path the integrand is well behaved the integration difficulties go away when $t$ is large enough.
[1] G. E. King, SPE Hydraulic Fracturing Technology Conference (OnePetro, 2012).

[2] L. Weijers, C. Wright, M. Mayerhofer, M. Pearson, L. Griffin, and P. Weddle, SPE Hydraulic Fracturing Technology Conference and Exhibition (OnePetro, 2019).

[3] M. Marder, T. Patzek, and S. W. Tinker, Phys. Today 69, 46 (2016).

[4] T. W. Patzek, W. Saputra, W. Kirati, and M. Marder, Energy Fuels 33, 12154 (2019).

[5] M. D. Zoback and A. H. Kohli, Unconventional Reservoir Geomechanics (Cambridge University Press, Cambridge, 2019).

[6] T. Patzek, F. Male, and M. Marder, Proc. Natl. Acad. Sci. USA 110, 19731 (2013).

[7] W. Saputra, W. Kirati, and T. Patzek, Energies 13, 2052 (2020).

[8] C. L. Cipolla, N. R. Warpinski, and M. J. Mayerhofer, SPE Asia Pacific Oil and Gas Conference and Exhibition (Society of Petroleum Engineers, 2008).

[9] P. Valkó, in Kirk-Othmer Encyclopedia of Chemical Technology (John Wiley \& Sons, 2014), pp. 1-24, doi: 10.1002/0471238961.hydrvalk.a01.

[10] K. T. Raterman, H. E. Farrell, O. S. Mora, A. L. Janssen, G. A. Gomez, S. Busetti, J. McEwen, M. Davidson, K. Friehauf, J. Rutherford et al., SPE Res. Eval. Eng. 21, 927 (2018).

[11] G. Jin and B. Roy, Leading Edge 36, 975 (2017).

[12] T. A. Witten Jr. and L. M. Sander, Phys. Rev. Lett. 47, 1400 (1981).

[13] M. Marder, B. Eftekhari, and T. W. Patzek, Phys. Rev. Lett. 120, 138302 (2018).

[14] B. Eftekhari, M. Marder, and T. W. Patzek, J. Nat. Gas Sci. Eng. 56, 141 (2018).

[15] B. Eftekhari, M. Marder, and T. Patzek, SPE Reservoir Eval. Eng. 23, 664 (2020).
[16] S. Haider, W. Saputra, and T. Patzek, Energies 13, 2348 (2020).

[17] B. Eftekhari, A lattice model for gas production from hydrofractured shale, Ph.D. thesis, University of Texas at Austin, 2016.

[18] W. Saputra, W. Kirati, and T. W. Patzek, J. Nat. Gas Sci. Eng. 94, 104041 (2021).

[19] E. Bonnet, O. Bour, N. E. Odling, P. Davy, I. Main, P. Cowie, and B. Berkowitz, Rev. Geophys. 39, 347 (2001).

[20] W. Zhu, S. Khirevich, and T. W. Patzek, Water Resour. Res. 57, e2020WR028652 (2021).

[21] E. N. Economou, Green's Functions in Quantum Physics (Springer-Verlag, Berlin, 1983).

[22] M. Newville, T. Stensitzki, D. Allen, A. Ingargiola, T. Stensitzki, and D. Allan, LMFIT: Non-Linear Least-Square Minimization and Curve-Fitting for Python (2021).

[23] T. Morita, J. Phys. A 8, 478 (1975).

[24] M. Berciu and A. M. Cook, Europhys. Lett. 92, 40003 (2010).

[25] F. Johansson et al., mpmath: A Python library for arbitraryprecision floating-point arithmetic (version 1.1.0) (2018), http://mpmath.org/.

[26] E. Jones, T. Oliphant, P. Peterson et al., SciPy: Open Source Scientific Tools for Python (2001), http://www.scipy.org/.

[27] G. Van Rossum and F. L. Drake, Jr, Python Tutorial (Centrum voor Wiskunde en Informatica, Amsterdam, 1995).

[28] F. Johansson, IEEE Trans. Comput. 66, 1281 (2017).

[29] D. M. Beazley, in Proceedings of the 4th Conference on USENIX Tcl/Tk Workshop, TCLTK'96 (USENIX Association, Berkeley, CA, 1996), Vol. 4, pp. 15-15.

[30] J. J. Dongarra, C. B. Moler, J. R. Bunch, and G. W. Stewart, LINPACK Users' Guide (SIAM, Philadelphia, 1979).

[31] R. Piessens, E. de Doncker-Kapenga, C. W. Überhuber, and D. K. Kahaner, Quadpack: A Subroutine Package for Automatic Integration (Springer Science \& Business Media, 2012), Vol. 1. 